TRANSACTIONS OF THE

AMERICAN MATHEMATICAL SOCIETY

Volume 366, Number 1, January 2014, Pages 505-530

S 0002-9947(2013)05894-1

Article electronically published on July 16, 2013

\title{
THE NONLINEAR HEAT EQUATION WITH HIGH ORDER MIXED DERIVATIVES OF THE DIRAC DELTA AS INITIAL VALUES
}

\author{
SLIM TAYACHI AND FRED B. WEISSLER
}

\begin{abstract}
In this paper we prove local existence of solutions of the nonlinear heat equation $u_{t}=\Delta u+|u|^{\alpha} u, t \in(0, T), x \in \mathbb{R}^{N}$, with initial value $u(0)=$ $K \partial_{1} \partial_{2} \cdots \partial_{m} \delta, K \neq 0, m \in\{1,2, \cdots, N\}, 0<\alpha<2 /(N+m)$ and $\delta$ is the Dirac distribution. In particular, this gives a local existence result with an initial value in a high order negative Sobolev space $H^{s, q}\left(\mathbb{R}^{N}\right)$ with $s \leq-2$.

As an application, we prove the existence of initial values $u_{0}=\lambda f$ for which the resulting solution blows up in finite time if $\lambda>0$ is sufficiently small. Here, $f$ satisfies in particular $f \in C_{0}\left(\mathbb{R}^{N}\right) \cap L^{1}\left(\mathbb{R}^{N}\right)$ and is anti-symmetric with respect to $x_{1}, x_{2}, \cdots, x_{m}$. Moreover, we require $\int_{\mathbb{R}^{N}} x_{1} \cdots x_{m} f(x) d x \neq 0$. This extends the known "small lambda" blow up results which require either that $\int_{\mathbb{R}^{N}} f(x) d x \neq 0$ (Dickstein (2006)) or $\int_{\mathbb{R}^{N}} x_{1} f(x) d x \neq 0$ (Ghoul (2011), (2012)).
\end{abstract}

\section{INTRODUCTION}

In this paper we study local existence and uniqueness of solutions to the semilinear heat equation in integral form,

$$
u(t)=\mathrm{e}^{t \Delta} u_{0}+\int_{0}^{t} \mathrm{e}^{(t-\sigma) \Delta}\left(|u(\sigma)|^{\alpha} u(\sigma)\right) d \sigma
$$

where $\mathrm{e}^{t \Delta}$ is the heat semigroup on $\mathbb{R}^{N}, \alpha>0$ and $u_{0} \in \mathcal{S}^{\prime}\left(\mathbb{R}^{N}\right)$ is a multiple of

$$
(-1)^{m} \partial_{1} \partial_{2} \cdots \partial_{m} \delta
$$

$1 \leq m \leq N$ and $\delta$ is the Dirac point mass at the origin. Recall that $\mathrm{e}^{t \Delta} f=G_{t} \star f$, where

$$
G_{t}(x)=(4 \pi t)^{-N / 2} \mathrm{e}^{-\frac{|x|^{2}}{4 t}}, t>0, x \in \mathbb{R}^{N} .
$$

For $x=\left(x_{1}, x_{2}, \cdots, x_{N}\right)$, we denote $\partial_{x_{i}}$ by $\partial_{i}, 1 \leq i \leq N$. We seek a solution $u \in C\left((0, T] ; C_{0}\left(\mathbb{R}^{N}\right)\right)$ such that $u(t) \rightarrow u_{0}$ in $\mathcal{S}^{\prime}\left(\mathbb{R}^{N}\right)$ as $t \rightarrow 0 . C_{0}\left(\mathbb{R}^{N}\right)$ denotes the set of continuous functions on $\mathbb{R}^{N}$ which tend to zero at infinity and $\mathcal{S}^{\prime}\left(\mathbb{R}^{N}\right)$ denotes the space of tempered distributions on $\mathbb{R}^{N}$.

It is well known that (1.1) is locally well posed in $C_{0}\left(\mathbb{R}^{N}\right)$. In particular, given any $u_{0} \in C_{0}\left(\mathbb{R}^{N}\right)$, there exist $T_{\max }\left(u_{0}\right)>0$ and a unique continuous solution

Received by the editors November 21, 2011 and, in revised form, May 29, 2012.

2010 Mathematics Subject Classification. Primary 35K55, 35A01, 35B44; Secondary 35K57, $35 \mathrm{C} 15$.

Key words and phrases. Nonlinear heat equation, highly singular initial values, finite time blow-up.

(C) 2013 American Mathematical Society Reverts to public domain 28 years from publication 
$u:\left[0, T_{\max }\left(u_{0}\right)\right) \rightarrow C_{0}\left(\mathbb{R}^{N}\right)$ of 1.1) with $u(0)=u_{0}$. This solution is a classical solution of

$$
u_{t}=\Delta u+|u|^{\alpha} u
$$

for $0<T<T_{\max }\left(u_{0}\right)$. Furthermore, if $T_{\max }\left(u_{0}\right)<\infty$, then $\|u(t)\|_{\infty} \rightarrow \infty$ as $t \rightarrow T_{\max }\left(u_{0}\right)$.

The equation (1.1) is also locally well posed in other function spaces. For example, if $\alpha<2 q / N$ and $q \geq 1$, then (1.1) is locally well posed in $L^{q}\left(\mathbb{R}^{N}\right)$. See [20, 21]. If $u_{0} \in L^{q}\left(\mathbb{R}^{N}\right)$, then the resulting solution is continuous $u:\left(0, T_{\max }\left(u_{0}\right)\right) \rightarrow$ $C_{0}\left(\mathbb{R}^{N}\right)$ and a classical solution of (1.4) for $0<T<T_{\max }\left(u_{0}\right)$. (Here we are not concerned with the limiting case $\alpha=2 q / N$ or the precise formulation of uniqueness in $L^{q}\left(\mathbb{R}^{N}\right)$.) In the case $q=1$ and $\alpha<2 / N$, (1.1) is also locally well posed in $\mathcal{M}$, the space of finite Radon measures. In particular, this includes the Dirac mass, i.e. example (1.2) in the case $m=0$.

In addition, (1.1) is locally well posed in certain negative order Sobolev spaces $H^{s, q}\left(\mathbb{R}^{N}\right)$, i.e. with $s<0$. See Ribaud [18, Wu [25, and Ghoul 7]. Without going into the details of these results, it suffices to remark that if $\alpha<1 /(N+1)$, then (1.1) is locally well posed in a negative Sobolev space which contains the distribution $\partial_{i} \delta$. See the proof of Theorem 18 in [7]. This is the case $m=1$ in (1.2).

The novelty of our methods is that we can treat the cases $2 \leq m \leq N$. Also, we improve the result in the case $m=1$ treated in [7. We emphasize that our methods do not enable us to prove that (1.1) is locally well posed in high negative order Sobolev spaces. Indeed, it seems there is an obstacle to such a result. If $s \leq-2$, then the semigroup part of the integral term is too singular to be handled in standard function spaces. The known results, for example Corollary 11 in [7, explicitly require $s>-2$. We can only handle very specific initial values in these spaces. For example, if $N=2$, we can prove the existence of solutions to (1.1) with initial value $u_{0}=\partial_{x y} \delta$ but not $u_{0}=\partial_{x x} \delta$ or $u_{0}=\partial_{y y} \delta$.

Our first main result is the following.

Theorem 1.1. Let the positive integer $m$ and the real number $\alpha$ be such that

$$
1 \leq m \leq N, 0<\alpha<\frac{2}{N+m} .
$$

Let $K \in \mathbb{R}, K \neq 0$, and set

$$
u_{0}=K(-1)^{m} \partial_{1} \partial_{2} \cdots \partial_{m} \delta .
$$

There exist $T>0$ and a continuous solution $u:(0, T] \rightarrow C_{0}\left(\mathbb{R}^{N}\right)$ of the integral equation (1.1) with initial value $u_{0}$ and such that $u(t) \rightarrow u_{0}$ in $\mathcal{S}^{\prime}\left(\mathbb{R}^{N}\right)$ as $t \rightarrow 0$.

The uniqueness part of Theorem[1.1] is rather technical to state here and is better understood in the following sections. See Theorem 2.3 and Proposition 3.3 .

It turns out that the values of $\alpha>0$, for which a solution exists with initial value given by (1.5), are precisely those predicted by a formal scaling argument. The initial value $\partial_{1} \partial_{2} \cdots \partial_{m} \delta$ is in the Sobolev space $H^{s, q}\left(\mathbb{R}^{N}\right)$ when $s<\frac{N}{q}-N-m$. On the other hand, the "scaling critical exponent $s_{c}$ " is given by $s_{c}=\frac{N}{q}-\frac{2}{\alpha}$. To see this, we observe that the transformation $\lambda^{\frac{2}{\alpha}} u\left(\lambda^{2} t, \lambda x\right)$, which leaves invariant the set of solutions to $u_{t}=\Delta u+|u|^{\alpha} u$, acts on initial values as $\lambda^{\frac{2}{\alpha}} u_{0}(\lambda \cdot)$. Furthermore,

$$
\left\|\lambda^{\frac{2}{\alpha}} u_{0}(\lambda \cdot)\right\|_{\dot{H}^{s, q}\left(\mathbb{R}^{N}\right)}=\lambda^{\frac{2}{\alpha}-\frac{N}{q}+s}\left\|u_{0}(\cdot)\right\|_{\dot{H}^{s, q}\left(\mathbb{R}^{N}\right)}, \forall \lambda>0 .
$$


In other words, the homogeneous Sobolev norm of $\dot{H}^{s, q}\left(\mathbb{R}^{N}\right)$ is invariant under the action of $\lambda^{\frac{2}{\alpha}} u_{0}(\lambda \cdot)$ precisely if $s=s_{c}$. We would "hope" that solutions exist if the initial value is in some $H^{s, q}\left(\mathbb{R}^{N}\right)$ with $s>s_{c}$. With the initial value given by (1.5), this happens when $\frac{N}{q}-N-m>\frac{N}{q}-\frac{2}{\alpha}$, i.e. $\alpha<2 /(N+m)$. We refer the reader to 2 for the definitions and basic properties of the Sobolev spaces $H^{s, q}\left(\mathbb{R}^{N}\right)$, the homogeneous Sobolev spaces $\dot{H}^{s, q}\left(\mathbb{R}^{N}\right)$, and the space of tempered distributions $\mathcal{S}^{\prime}\left(\mathbb{R}^{N}\right)$.

Our method to prove Theorem [1.1, going back to Fujita [6] and an earlier work of the second author [22, is based on the following idea: one looks for solutions $u$ such that $u(t) \leq C \mathrm{e}^{t \Delta} u_{0}$. Fujita [6] used this idea to show, in the case $\alpha>2 / N$, that if $u_{0}$ is bounded by a small enough Gaussian, then the resulting solution of (1.1) is global in time. In [22, this idea was used to find new sufficient conditions on $u_{0}$ which imply that the resulting solution of (1.1) is global. In this paper, we give a more general version of the arguments in [22] and apply them to the heat semigroup on domains of the form

$$
\Omega=\left\{x \in \mathbb{R}^{N}: x_{1}>0, x_{2}>0, \cdots, x_{m}>0\right\} .
$$

Functions in $C_{0}\left(\mathbb{R}^{N}\right)$, which are anti-symmetric in $x_{1}, x_{2}, \cdots, x_{m}$, restrict to functions in $C_{0}(\Omega)$, and functions in $C_{0}(\Omega)$ extend uniquely to functions in $C_{0}\left(\mathbb{R}^{N}\right)$ which are anti-symmetric in $x_{1}, x_{2}, \cdots, x_{m}$. This enables us to prove the existence of solutions to (1.1) in $C_{0}(\Omega)$, with the appropriate heat semigroup, and then consider them as solutions in $C_{0}\left(\mathbb{R}^{N}\right)$ which are anti-symmetric in $x_{1}, x_{2}, \cdots, x_{m}$. Solutions with initial values $u_{0}$ of the form (1.5) fit precisely into this situation, as can be seen by looking at $\mathrm{e}^{t \Delta} u_{0}$. The solutions we construct will be positive (or negative, depending on the sign of $K$ ) on $\Omega$.

Furthermore, the solution $u:(0, T] \rightarrow C_{0}\left(\mathbb{R}^{N}\right)$ constructed as above can be extended to a maximal solution of (1.1) $, u:\left(0, T_{\max }\right) \rightarrow C_{0}\left(\mathbb{R}^{N}\right)$ with $u_{0}$ given by (1.5). Since these solutions have a definite sign on $\Omega$ it follows from the well-known Fujita-type results on sectors (see [16, 13, 1, 15]) that $T_{\max }<\infty$.

In principle, our method should apply more generally, for other highly singular initial values such as

$$
u_{0}=K(-1)^{m} \partial_{1} \partial_{2} \cdots \partial_{m} f
$$

for certain $f \in L^{1}\left(\mathbb{R}^{N}\right)+L^{q}\left(\mathbb{R}^{N}\right)$. But for the moment, such a result eludes us.

Aside from being of interest in its own right, the existence of solutions to (1.1) with initial values of the form (1.2) has important implications for the finite time blow up properties of solutions with regular initial values. The relationship we have in mind was discovered by Dickstein [4. Suppose $f \in L^{1}\left(\mathbb{R}^{N}\right) \cap C_{0}\left(\mathbb{R}^{N}\right)$ and $\int_{\mathbb{R}^{N}} f \neq 0$ and that $\alpha<2 / N$ (the Fujita exponent). It follows that $T_{\max }(\lambda f)<\infty$ for all sufficiently small $\lambda>0$. This extends Fujita's result [6], for the same values of $\alpha$, that $T_{\max }\left(u_{0}\right)<\infty$ for all positive $u_{0} \in C_{0}\left(\mathbb{R}^{N}\right)$. Dickstein's proof is based on a rescaling of $\lambda f$ as $u_{0}^{\lambda, \mu}=\lambda \mu^{2 / \alpha} f(\mu \cdot)$, with $\mu>0$ chosen so that $u_{0}^{\lambda, \mu} \rightarrow c \delta$ as $\lambda \rightarrow 0$, where $c=\int_{\mathbb{R}^{N}} f \neq 0$. Since the solution with initial value $c \delta$ blows up in finite time (by Fujita's result), Dickstein is able to show the same for $u_{0}^{\lambda, \mu}$, and thus $\lambda f$, for sufficiently small $\lambda>0$.

Recently, Ghoul [7] has shown that if $\alpha<1 /(N+1)$, the condition $\int_{\mathbb{R}^{N}} f \neq 0$ can be replaced by the condition $\int_{\mathbb{R}^{N}} f=0$, but $f=\partial_{i} g$, where $g \in L^{1}\left(\mathbb{R}^{N}\right)$ and 
$\int_{\mathbb{R}^{N}} g \neq 0$. Ghoul uses a different rescaling of the initial value so that the rescaled initial value converges to a nonzero multiple of $\partial_{i} \delta$. Ghoul's result mentioned above that (1.1) is well posed in certain negative order Sobolev spaces which contain $\partial_{i} \delta$ leads to the same conclusion that $T_{\max }(\lambda f)<\infty$ for all sufficiently small $\lambda>0$. For this, Ghoul needs to use the Fujita-type result on a half space due to Meier [16], Bandle and Levine [1, Levine and Meier [15, and Kavian [13.

Our results on existence of solutions to (1.1) with initial values of the form (1.2) suggest that a further extension of Dickstein's result is possible. Indeed we establish such a result under the condition $\alpha<2 /(N+m)$. More precisely, we prove the following.

Theorem 1.2. Let the positive integer $m$ and the real number $\alpha$ be such that

$$
1 \leq m \leq N, 0<\alpha<\frac{2}{N+m} .
$$

Let $f \in C_{0}\left(\mathbb{R}^{N}\right)$ be anti-symmetric with respect to $x_{1}, x_{2}, \cdots, x_{m}$, and suppose that there exist $t_{0}>0$ and $K>0$ such that

$$
|f(x)| \leq K\left|\partial_{1} \partial_{2} \cdots \partial_{m} G_{t_{0}}(x)\right|, \forall x \in \mathbb{R}^{N} .
$$

Moreover, assume that

$$
K_{0} \equiv \int_{\mathbb{R}^{N}} x_{1} x_{2} \cdots x_{m} f(x) d x_{1} d x_{2} \cdots d x_{N} \neq 0
$$

It follows that the maximal solution $u:\left[0, T_{\max }\right) \rightarrow C_{0}\left(\mathbb{R}^{N}\right)$ of the integral equation (1.1) with initial value $u_{0}=\lambda f$ blows up in finite time if $\lambda>0$ is sufficiently small.

See Proposition 4.1 for additional properties of a function $f$ satisfying the above conditions, and Remark 4.2 for the existence of such functions.

It is the assumption $K_{0} \neq 0$ which replaces Dickstein's assumption that the initial value has nonzero mean value. Here, we clearly have $\int_{\mathbb{R}^{N}} u_{0}=0$ since $u_{0}$ is anti-symmetric with respect to at least one variable. Also, the case $m=1$ was treated by Ghoul [7. The hypothesis on $u_{0}$ needed in [7] is formally equivalent (and in many cases genuinely equivalent) to the condition $K_{0} \neq 0$ with $m=1$. See Corollary 1 in [8] and its proof. Thus, our result is a genuine extension of those in [4, 7].

Also, while $(-1)^{m} \partial_{1} \partial_{2} \cdots \partial_{m} G_{t_{0}}(x)>0$ for all $x \in \Omega$, we most definitely do not make that assumption for $f$. This is the whole point of Theorem 1.2. If $f>0$ on $x \in \Omega$, then the solution with initial value $\lambda f$ would blow up in finite time for all $\lambda>0$ because of the Fujita-type results on $\Omega$ in [16, 13, 1, 15]. In addition, we note that the condition $\alpha<\frac{2}{N+m}$ in Theorem 1.2 is sharp. If $\alpha>\frac{2}{N+m}$, then all initial data antisymmetric in $x_{1}, x_{2}, \cdots, x_{m}$ and satisfying (1.7) for some $t_{0}>0$ and sufficiently small $K$ produce solutions which are global in time. See Remark 4.3 below.

To prove Theorem 1.2, we use a dilation for which the rescaled initial value converges to a nonzero multiple of (1.2), i.e. (1.5). As already noted, the maximal solution with initial value (1.5) blows up in finite time. Since we do not prove well posedness of (1.1) in any standard function space, we need to provide an ad hoc proof that the solutions with the rescaled initial values converge in $C_{0}\left(\mathbb{R}^{N}\right)$ at some positive time to the solution with initial value of the form (1.5). 
If $f$ is as in the statement of Theorem 1.2 and also in $H^{1}\left(\mathbb{R}^{N}\right)$, then by standard energy arguments 14, the maximal solution $u:\left[0, T_{\max }\right) \rightarrow C_{0}\left(\mathbb{R}^{N}\right)$ of the integral equation (1.1) with initial value $u_{0}=\lambda f$ blows up in finite time if $\lambda>0$ is sufficiently large as well. Thus, it would be interesting to find such a function so that the solution with initial value $u_{0}=f$, i.e. with $\lambda=1$, is in fact global. For such a function $f$, the solution with initial value $u_{0}=\lambda f$ would blow up in finite time for large and small $\lambda>0$, but it would be global for $\lambda=1$. Such a result was proved in the context of Dickstein's small lambda theorem [4, i.e. in the case $m=0$ and with the requirement that $\int_{\mathbb{R}^{N}} f d x \neq 0$, in the paper 3 . A similar result was proved in the case $m=1$ in [8]. This result allows $\int_{\mathbb{R}^{N}} f d x=0$, but requires $\int_{\mathbb{R}^{N}} x_{j} f d x \neq 0$ for some $1 \leq j \leq N$. Here, we obtain such a result in the case $m=2$. We allow both $\int_{\mathbb{R}^{N}} f d x=0$ and $\int_{\mathbb{R}^{N}} x_{j} f d x=0$, but require $\int_{\mathbb{R}^{N}} x_{j} x_{k} f d x \neq 0$ for some $j \neq k$. Unfortunately, it is not clear how our proof could extend to general $m$, with $2 \leq m \leq N$, since that would require higher order regularity of the solution, which is unlikely for $\alpha<1$.

Theorem 1.3. Let the real number $\alpha$ be such that

$$
0<\alpha<\frac{2}{N+2} .
$$

There exists a function $f \in C_{0}\left(\mathbb{R}^{N}\right) \cap H^{1}\left(\mathbb{R}^{N}\right)$, verifying the hypotheses of Theorem 1.2 in the case $m=2$, such that the maximal solution $u:\left[0, T_{\max }\right) \rightarrow C_{0}\left(\mathbb{R}^{N}\right)$ of the integral equation (1.1) with initial value $u_{0}=f$ is global in time.

The rest of this paper is organized as follows. In Section 2, we establish the local existence of solutions to an integral equation in an abstract setting. This result does not give information about the initial value. In Section 3 we first use the result in Section 2 to prove the existence of solutions to equation (1.1) with $u_{0}$ given by (1.5). We also provide a proof that $u(t) \rightarrow u_{0}$ in $\mathcal{S}^{\prime}\left(\mathbb{R}^{N}\right)$ as $t \rightarrow 0$. In Section 4 we establish the blow up result, Theorem 1.2, and in Section 5 we establish the global existence result, Theorem 1.3 .

\section{LOCAL EXISTENCE IN A GENERAL CONTEXT}

Let $\Omega \subset \mathbb{R}^{N}, N \geq 1$, be a regular domain not necessarily bounded. The Banach space $C_{0}(\Omega)$ is the set of all continuous functions $f: \bar{\Omega} \rightarrow \mathbb{R}$ such that $f=0$ on the boundary $\partial \Omega$ and $f(x) \rightarrow 0$ as $|x| \rightarrow \infty$ in $\Omega$. The sup norm on $C_{0}(\Omega)$ is designated by $\|f\|_{\infty}$. We assume that $\Omega$ is regular enough so that the heat semigroup $\mathrm{e}^{t \Delta}$ is a $C_{0}$ contraction, positivity preserving semigroup on $C_{0}(\Omega)$. Since $\mathrm{e}^{t \Delta}$ is positivity preserving, it satisfies the inequality

$$
\left|\mathrm{e}^{t \Delta}(f g)\right| \leq\left(\mathrm{e}^{t \Delta}|f|\right)\|g\|_{\infty},
$$

for all $f, g \in C_{0}(\Omega)$, for example.

Definition 2.1. A continuous function $\varphi:(0, \infty) \rightarrow C_{0}(\Omega)$ is called a solution of the linear heat equation if

$$
\mathrm{e}^{t \Delta} \varphi(s)=\varphi(t+s)
$$

for all $t>0$ and $s>0$. 
It is important to note that no initial value $\varphi(0)$ is required or specified. Also, note that if $\varphi:(0, \infty) \rightarrow C_{0}(\Omega)$ is a solution of the linear heat equation, then $\|\varphi(t)\|_{\infty}$ is a nonincreasing function of $t>0$.

Definition 2.2. Let $\Phi:(0, \infty) \rightarrow C_{0}(\Omega)$ be a positive solution of the linear heat equation, i.e. $\Phi(t, x)>0$ for all $t>0, x \in \Omega$. For a given $T>0$, let $X=X_{T}=X_{T, \Phi}$ be the set of all continuous functions $u:(0, T] \rightarrow C_{0}(\Omega)$ which are bounded by a multiple of $\Phi$ in that there exists $M>0$ such that

$$
|u(t, x)| \leq M \Phi(t, x), \forall t \in(0, T], \forall x \in \Omega .
$$

We define on $X$ the norm

$$
\|\mid u\|=\sup _{0<t \leq T, x \in \Omega} \frac{|u(t, x)|}{\Phi(t, x)} .
$$

The set $X$ equipped with this norm is a Banach space. Note that

$$
|u(t)| \leq \||| u|| \mid \Phi(t)
$$

for all $0<t \leq T$. If needed for clarity, we may denote the norm \|\|$u \|||$ by $\||u|\|_{X_{T}}$ or even $\||| u \mid\|_{X_{T, \Phi}}$.

We have the following result.

Theorem 2.3. Fix a positive solution of the linear heat equation $\Phi:(0, \infty) \rightarrow$ $C_{0}(\Omega)$. Let $\alpha>0$, and suppose that $\Phi$ verifies

$$
\int_{0}^{A}\|\Phi(\sigma)\|_{\infty}^{\alpha} d \sigma<\infty
$$

for some (hence all) $A>0$. Let $K>0, M>0$ and $T>0$ be such that

$$
K+M^{\alpha+1} \int_{0}^{T}\|\Phi(t)\|_{\infty}^{\alpha} d t \leq M
$$

and

$$
2(\alpha+1) M^{\alpha} \int_{0}^{T}\|\Phi(t)\|_{\infty}^{\alpha} d t<1 .
$$

Given $\varphi \in X_{T}$ with $\|\varphi\| \| \leq K$, there is a unique solution $u \in X_{T}$ with \|\|$u \| \mid \leq M$ of the integral equation

$$
u(t)=\varphi(t)+\int_{0}^{t} \mathrm{e}^{(t-\sigma) \Delta}\left(|u(\sigma)|^{\alpha} u(\sigma)\right) d \sigma .
$$

Moreover, the integral in (2.6) is absolutely convergent in $C_{0}(\Omega)$, and

$$
\lim _{t \rightarrow 0}\left\|\frac{u(t)-\varphi(t)}{\Phi(t)}\right\|_{\infty}=0 .
$$

Furthermore, there exists $\mathcal{C}>0$ such that if $\varphi \in X_{T}$ and $\psi \in X_{T}$, with $\|\varphi\| \| \leq K$ and $\||\psi \|| \leq K$, and if $u$ and $v$ are respectively the solutions of the corresponding integral equations, then

$$
\|u-v\|\|\leq \mathcal{C}\| \mid \varphi-\psi\|\| .
$$

Finally, if $\varphi \in X_{T}$ with $\|\varphi \varphi\| \leq K$ is positive, $\varphi(t) \geq 0$, for all $0<t \leq T$, then the same is true of the resulting solution $u \in X_{T}$ of (2.6) with $\|u\| \| \leq M$, i.e. $u(t) \geq 0$ for all $0<t \leq T$. 
Remark 2.4. The previous theorem gives a local existence result of solutions for (1.1) with $u_{0} \in L^{q}\left(\mathbb{R}^{N}\right), q \geq 1, q>\frac{N \alpha}{2}$. In fact, by the smoothing properties of the semigroup of the linear heat equation, we have that $\left\|\mathrm{e}^{t \Delta}\left|u_{0}\right|\right\|_{L^{\infty}(\Omega)} \leq t^{-\frac{N}{2 q}}\left\|u_{0}\right\|_{L^{q}(\Omega)}$. Then we apply Theorem 2.3 with $\varphi(t)=\mathrm{e}^{t \Delta} u_{0}, \Phi(t)=\mathrm{e}^{t \Delta}\left|u_{0}\right|$ and $K=1$. Clearly, by the conditions on $q,(2.3)$ is satisfied. This result, of course, is well known [20, 21, 17.

Remark 2.5. We emphasize that Theorem 2.3 neither requires nor mentions an initial value to the integral equation (2.6). Moreover, the function $\varphi$ need not itself be a solution of the linear heat equation.

Proof of Theorem 2.3. The proof is based on a contraction mapping argument, and is inspired by the construction in 22. We thank Thierry Cazenave for pointing out how the construction in 22 could be reformulated as a contraction mapping argument. Moreover, a similar argument was used by Fujita 6] to prove global existence of solutions for initial values bounded by a small Gaussian function.

Let $K>0, M>0$ and $T>0$ satisfying (2.4) and (2.5). Let

$$
B(0, M):=\{u \in X|\|u\|| \leq M\} .
$$

$B(0, M)$ is a complete metric space. Fix $\varphi \in X$ such that \|\|$\varphi\|\| \leq K$ and consider, for $u \in B(0, M)$,

$$
\mathcal{F} u(t)=\varphi(t)+\int_{0}^{t} \mathrm{e}^{(t-\sigma) \Delta}\left(|u(\sigma)|^{\alpha} u(\sigma)\right) d \sigma
$$

We will show that $\mathcal{F}$ is a strict contraction on $B(0, M)$.

First, using the inequalities (2.1) and (2.2), we have that

$$
\begin{aligned}
|\mathcal{F} u(t)| & \leq|\varphi(t)|+\int_{0}^{t}\left(\mathrm{e}^{(t-\sigma) \Delta}|u(\sigma)|\right)\|u(\sigma)\|_{\infty}^{\alpha} d \sigma \\
& \leq K \Phi(t)+\|\| u\left\|\left.\right|^{\alpha+1} \int_{0}^{t}\left(\mathrm{e}^{(t-\sigma) \Delta} \Phi(\sigma)\right)\right\| \Phi(\sigma) \|_{\infty}^{\alpha} d \sigma \\
& \leq K \Phi(t)+M^{\alpha+1} \int_{0}^{t} \Phi(t)\|\Phi(\sigma)\|_{\infty}^{\alpha} d \sigma \\
& \leq \Phi(t)\left[K+M^{\alpha+1} \int_{0}^{T}\|\Phi(\sigma)\|_{\infty}^{\alpha} d \sigma\right] .
\end{aligned}
$$

Hence,

$$
\frac{|\mathcal{F} u(t)|}{\Phi(t)} \leq K+M^{\alpha+1} \int_{0}^{T}\|\Phi(\sigma)\|_{\infty}^{\alpha} d \sigma, \forall t \in(0, T] .
$$

Thus by (2.4), we have that || $\mathcal{F} u \mid \| \leq M$, and so $\mathcal{F}$ maps $B(0, M)$ into itself.

Next, to see that $\mathcal{F}$ is a contraction, we let $u_{1}, u_{2} \in B(0, M)$ so that

$$
\mathcal{F}\left(u_{1}\right)(t)-\mathcal{F}\left(u_{2}\right)(t)=\int_{0}^{t} \mathrm{e}^{(t-\sigma) \Delta}\left(\left|u_{1}\right|^{\alpha} u_{1}(\sigma)-\left|u_{2}\right|^{\alpha} u_{2}(\sigma)\right) d \sigma .
$$


It follows that

$$
\begin{aligned}
& \left|\mathcal{F}\left(u_{1}\right)(t)-\mathcal{F}\left(u_{2}\right)(t)\right| \\
& \leq(\alpha+1) \int_{0}^{t} \mathrm{e}^{(t-\sigma) \Delta}\left(\left|u_{1}(\sigma)-u_{2}(\sigma)\right|\left(\left|u_{1}(\sigma)\right|^{\alpha}+\left|u_{2}(\sigma)\right|^{\alpha}\right)\right) d \sigma \\
& \leq(\alpha+1) \int_{0}^{t}\left(\mathrm{e}^{(t-\sigma) \Delta} \Phi(\sigma) \frac{\left|u_{1}(\sigma)-u_{2}(\sigma)\right|}{\Phi(\sigma)}\right) \\
& \quad\left(\left\|u_{1}(\sigma)\right\|_{\infty}^{\alpha}+\left\|u_{2}(\sigma)\right\|_{\infty}^{\alpha}\right) d \sigma \\
& \leq(\alpha+1) \int_{0}^{t}\left(\mathrm{e}^{(t-\sigma) \Delta} \Phi(\sigma)\right)\left\|\left|u_{1}-u_{2}\right|\right\| \\
& \left.\quad \leq 2 M^{\alpha}(\alpha+1) \int_{0}^{t} \Phi(t)\|\Phi(\sigma)\|_{\infty}^{\alpha} d \sigma\left\|u_{1}(\sigma)\right\|_{\infty}^{\alpha}+\left\|u_{1}-u_{2}(\sigma)\right\|_{\infty}^{\alpha}\right) d \sigma .
\end{aligned}
$$

Therefore,

$$
\frac{\left|\mathcal{F}\left(u_{1}\right)(t)-\mathcal{F}\left(u_{2}\right)(t)\right|}{\Phi(t)} \leq\left(2(\alpha+1) M^{\alpha} \int_{0}^{T}\|\Phi(\sigma)\|_{\infty}^{\alpha} d \sigma\right)\|\| u_{1}-u_{2}\|\|,
$$

and so by (2.5), there exists $\nu<1$ such that

$$
\left\|\left|\mathcal{F}\left(u_{1}\right)-\mathcal{F}\left(u_{2}\right)\right|\right\| \leq \nu||\left|u_{1}-u_{2}\right||| .
$$

Hence $\mathcal{F}$ is a strict contraction from $B(0, M)$ into itself. By the Banach fixed point theorem there exists a unique solution $u \in X_{T}$ of the integral equation (2.6) such that \|\|$u \| \mid \leq M$.

Let $u$ be the solution of the integral equation constructed above. Since

$$
\begin{aligned}
|u(t)-\varphi(t)| & =\left.\left|\int_{0}^{t} \mathrm{e}^{(t-\sigma) \Delta}\right| u(\sigma)\right|^{\alpha} u(\sigma) d \sigma \mid \\
& \leq \int_{0}^{t}\left(\mathrm{e}^{(t-\sigma) \Delta}|u(\sigma)|\right)\|u(\sigma)\|_{\infty}^{\alpha} d \sigma \\
& \leq M^{\alpha+1} \Phi(t) \int_{0}^{t}\|\Phi(\sigma)\|_{\infty}^{\alpha} d \sigma,
\end{aligned}
$$

assertion (2.7) follows. In the same way,

$$
\int_{0}^{t}\left\|\mathrm{e}^{(t-\sigma) \Delta}|u(\sigma)|^{\alpha} u(\sigma)\right\|_{\infty} d \sigma \leq M^{\alpha+1}\|\Phi(t)\|_{\infty} \int_{0}^{t}\|\Phi(\sigma)\|_{\infty}^{\alpha} d \sigma,
$$

which shows that the integral in (2.6) is absolutely convergent in $C_{0}(\Omega)$.

Let $\varphi \in X_{T}$ and $\psi \in X_{T}$ with $\|\phi\| \mid \leq K$ and \|\|$\psi \| \leq K$. Let $u$ and $v$ be the solutions of the corresponding integral equations, i.e.

$$
u(t)=\varphi(t)+\int_{0}^{t} \mathrm{e}^{(t-\sigma) \Delta}|u(\sigma)|^{\alpha} u(\sigma) d \sigma
$$

and

$$
v(t)=\psi(t)+\int_{0}^{t} \mathrm{e}^{(t-\sigma) \Delta}|v(\sigma)|^{\alpha} v(\sigma) d \sigma .
$$


Then, by a calculation similar to the proof above that $\mathcal{F}$ is a contraction, we have that

$$
\||u-v|\| \leq\left\|\left|\varphi-\psi\left\|\mid+\left(2(\alpha+1) M^{\alpha} \int_{0}^{T}\|\Phi(\sigma)\|_{\infty}^{\alpha} d \sigma\right)\right\|\|u-v\| \| .\right.\right.
$$

Thus (2.8) follows with

$$
\mathcal{C}=\frac{1}{1-\left(2(\alpha+1) M^{\alpha} \int_{0}^{T}\|\Phi(\sigma)\|_{\infty}^{\alpha} d \sigma\right)} .
$$

Finally, if $\varphi(t) \geq 0$ for all $0<t \leq T$, then one can carry out the contraction mapping argument on the subset of positive elements of $B(0, M)$.

Remark 2.6. If in the hypothesis of Theorem 2.3 we replace the condition (2.3) by

$$
\int_{0}^{\infty}\|\Phi(\sigma)\|_{\infty}^{\alpha} d \sigma<\infty
$$

and choose $K>0, M>0$ such that

$$
K+M^{\alpha+1} \int_{0}^{\infty}\|\Phi(t)\|_{\infty}^{\alpha} d t \leq M, \quad 2(\alpha+1) M^{\alpha} \int_{0}^{\infty}\|\Phi(t)\|_{\infty}^{\alpha} d t<1,
$$

then, if $\varphi \in X_{\infty}$ satisfies $\||\varphi|\|_{X_{\infty}} \leq K$, there exists a unique global solution to the integral equation (2.6), $u \in X_{\infty}$ with $\||u|\|_{X_{\infty}} \leq M$.

Remark 2.7. In the proof of Theorem 2.3 we only need that $\Phi:(0, T) \rightarrow C_{0}(\Omega)$ is positive and satisfies

$$
\mathrm{e}^{t \Delta} \Phi(s) \leq \Phi(t+s), \quad \forall t>0, s>0, \quad s+t<T .
$$

In other words, $\Phi$ could be a "super solution" of the linear heat equation. In this case, we define $\|\mid u\| \|=\sup _{0<t<T, x \in \Omega} \frac{|u(t, x)|}{\Phi(t, x)}$. This allows, in principle, that $\|\Phi(t)\|_{\infty} \rightarrow \infty$ as $t \rightarrow T$. Of course the resulting solution is only defined for $t \in$ $(0, T)$.

\section{Highly Singular initial VAlues}

The goal of this section is to use the abstract framework of the previous section as a tool to construct a solution of the nonlinear heat equation (1.4) on $\mathbb{R}^{N}$ with initial value $u_{0}$, which is an arbitrary given multiple of

$$
(-1)^{m} \partial_{1} \partial_{2} \cdots \partial_{m} \delta
$$

where $\delta \in \mathcal{S}^{\prime}\left(\mathbb{R}^{N}\right)$ is the Dirac point mass at the origin and $1 \leq m \leq N$. The solution of the linear heat equation on $\mathbb{R}^{N}$ with initial value (3.1) is well known. Recall that $G_{t}$, given by (1.3), is the Gauss (heat semigroup) kernel, and that $G_{t}$ is the solution of the linear heat equation on $C_{0}\left(\mathbb{R}^{N}\right)$ with initial value $\delta$. It follows that

$$
\Phi_{0}(t)=(-1)^{m} \partial_{1} \partial_{2} \cdots \partial_{m} G_{t}, t>0, x \in \mathbb{R}^{N},
$$

is the solution of the linear heat equation on $C_{0}\left(\mathbb{R}^{N}\right)$ with initial value (3.1). However, it is clearly not positive everywhere.

The key (albeit elementary) observation is that $\Phi_{0}(t)$ is a positive solution of the linear heat equation, as in Definition 2.1, in $C_{0}(\Omega)$, where

$$
\Omega=\mathbb{R}_{m^{+}}^{N}=\left\{x=\left(x_{1}, x_{2}, \cdots, x_{N}\right) \in \mathbb{R}^{N} \mid x_{1}>0, x_{2}>0, \cdots, x_{m}>0\right\} .
$$


Moreover, viewed as an element of $C_{0}\left(\mathbb{R}^{N}\right), \Phi_{0}(t)$ is anti-symmetric with respect to the variables $x_{1}, x_{2}, \cdots, x_{m}$. Indeed, by a simple calculation, we have that

$$
\Phi_{0}(t, x)=(4 \pi t)^{-\frac{N}{2}}(2 t)^{-m} \mathrm{e}^{-\frac{|x|^{2}}{4 t}} \prod_{i=1}^{m} x_{i} .
$$

It is clear that $\Phi_{0}(t, x)>0$ for all $x \in \Omega$, and that $\Phi_{0}(t) \in C_{0}(\Omega)$ for all $t>0$.

In order to put this observation in an appropriate context, we need to be more precise about the relationship between solutions of the linear and nonlinear equations in $C_{0}\left(\mathbb{R}^{N}\right)$ and in $C_{0}(\Omega)$. Throughout this section, $\Omega$ will be as in (3.3).

The first step is to identify the heat semigroup on $C_{0}(\Omega)$. As noted in the introduction, $\mathrm{e}^{t \Delta}$ is the heat semigroup on $\mathbb{R}^{N}$, i.e. convolution with the Gauss kernel (1.3), i.e.

$$
\mathrm{e}^{t \Delta} f=G_{t} \star f
$$

for all (for example) $f \in C_{0}\left(\mathbb{R}^{N}\right)$. We denote by $\mathrm{e}^{t \Delta_{\Omega}}$ the heat semigroup on $C_{0}(\Omega)$.

For $1 \leq i \leq N$, let $T_{i}: C_{0}\left(\mathbb{R}^{N}\right) \rightarrow C_{0}\left(\mathbb{R}^{N}\right)$ be the operator defined by

$$
\left[T_{i} f\right]\left(x_{1}, \cdots, x_{i-1}, x_{i}, x_{i+1}, \cdots, x_{N}\right)=f\left(x_{1}, \cdots, x_{i-1},-x_{i}, x_{i+1}, \cdots, x_{N}\right) .
$$

If $f \in C_{0}\left(\mathbb{R}^{N}\right)$ satisfies

$$
T_{1} f=T_{2} f=\cdots=T_{m} f=-f,
$$

then $f$ restricted to $\bar{\Omega}$ is clearly in $C_{0}(\Omega),\left.f\right|_{\bar{\Omega}} \in C_{0}(\Omega)$. On the other hand, if $g \in C_{0}(\Omega)$, then clearly $g$ can be extended uniquely to $\tilde{g} \in C_{0}\left(\mathbb{R}^{N}\right)$ so that $g=\left.\tilde{g}\right|_{\bar{\Omega}}$ and

$$
T_{1} \tilde{g}=T_{2} \tilde{g}=\cdots=T_{m} \tilde{g}=-\tilde{g} .
$$

It is immediate that $\mathrm{e}^{t \Delta} T_{i}=T_{i} \mathrm{e}^{t \Delta}$ for all $1 \leq i \leq m$. Consequently, if $g \in C_{0}(\Omega)$ and $\tilde{g} \in C_{0}\left(\mathbb{R}^{N}\right)$ is the extension of $g$ just defined, then

$$
T_{i} \mathrm{e}^{t \Delta} \tilde{g}=\mathrm{e}^{t \Delta} T_{i} \tilde{g}=-\mathrm{e}^{t \Delta} \tilde{g}
$$

for all $1 \leq i \leq m$. In other words, $\left.\left(\mathrm{e}^{t \Delta} \tilde{g}\right)\right|_{\bar{\Omega}} \in C_{0}(\Omega)$. We claim that the heat semigroup on $C_{0}(\Omega)$, denoted $\mathrm{e}^{t \Delta_{\Omega}}$, is given by

$$
\mathrm{e}^{t \Delta \Omega} g=\left.\left(\mathrm{e}^{t \Delta} \tilde{g}\right)\right|_{\bar{\Omega}},
$$

where $\mathrm{e}^{t \Delta}$ is the heat semigroup on $C_{0}\left(\mathbb{R}^{N}\right)$ and $\tilde{g}$ and $g$ are related as above. In fact, $\mathrm{e}^{t \Delta_{\Omega}} g$ is the solution of the linear heat equation on $C_{0}(\Omega)$ with initial value $g$. On the other hand, $\mathrm{e}^{t \Delta} \tilde{g}$ is the solution of the linear heat equation on $C_{0}\left(\mathbb{R}^{N}\right)$ with initial value $\tilde{g}$. Since $g=\left.\tilde{g}\right|_{\bar{\Omega}}$, (3.6) follows by uniqueness of solutions for the linear heat equations on $C_{0}(\Omega)$. In fact, the explicit expression for $\mathrm{e}^{t \Delta_{\Omega}} g$ is well known.

Proposition 3.1. The heat semigroup $\mathrm{e}^{t \Delta_{\Omega}}$ on $C_{0}(\Omega)$ is given by

$$
\mathrm{e}^{t \Delta_{\Omega}} g(x)=(4 \pi t)^{-\frac{N}{2}} \int_{\Omega} \prod_{j=m+1}^{N} \mathrm{e}^{-\frac{\left|x_{j}-y_{j}\right|^{2}}{4 t}} \prod_{i=1}^{m}\left[\mathrm{e}^{-\frac{\left|x_{i}-y_{i}\right|^{2}}{4 t}}-\mathrm{e}^{-\frac{\left|x_{i}+y_{i}\right|^{2}}{4 t}}\right] g(y) d y,
$$

for all $g \in C_{0}(\Omega)$.

Proof. First, let $N=1=m$. Hence $\Omega=] 0, \infty\left[\right.$. Assume that $g \in C_{0}(] 0, \infty[)$. Then $\tilde{g} \in C_{0}(\mathbb{R})$ and

$$
\tilde{g}(x)= \begin{cases}g(x) & \text { if } x>0 \\ -g(-x) & \text { if } x<0\end{cases}
$$


This gives

$$
\begin{aligned}
\mathrm{e}^{t \Delta} \tilde{g}(x) & =\int_{\mathbb{R}} G_{t}(x-y) \tilde{g}(y) d y \\
& =(4 \pi t)^{-\frac{1}{2}} \int_{\mathbb{R}} \mathrm{e}^{-\frac{|x-y|^{2}}{4 t}} \tilde{g}(y) d y \\
& =(4 \pi t)^{-\frac{1}{2}} \int_{0}^{\infty} \mathrm{e}^{-\frac{|x-y|^{2}}{4 t}} g(y) d y+(4 \pi t)^{-\frac{1}{2}} \int_{-\infty}^{0} \mathrm{e}^{-\frac{|x-y|^{2}}{4 t}}(-g(-y)) d y \\
& =(4 \pi t)^{-\frac{1}{2}} \int_{0}^{\infty} \mathrm{e}^{-\frac{|x-y|^{2}}{4 t}} g(y) d y-(4 \pi t)^{-\frac{1}{2}} \int_{0}^{\infty} \mathrm{e}^{-\frac{|x+y|^{2}}{4 t}} g(y) d y \\
& =\int_{0}^{\infty}(4 \pi t)^{-\frac{1}{2}}\left[\mathrm{e}^{-\frac{|x-y|^{2}}{4 t}}-\mathrm{e}^{-\frac{|x+y|^{2}}{4 t}}\right] g(y) d y
\end{aligned}
$$

Hence, for $x \in \Omega$,

$$
\mathrm{e}^{t \Delta_{\Omega}} g(x)=\int_{0}^{\infty}(4 \pi t)^{-\frac{1}{2}}\left[\mathrm{e}^{-\frac{|x-y|^{2}}{4 t}}-\mathrm{e}^{-\frac{|x+y|^{2}}{4 t}}\right] g(y) d y .
$$

Now, for $N \geq 1$, since the function $G_{t}(x)$ satisfies

$$
G_{t}(x)=(4 \pi t)^{-\frac{N}{2}} \prod_{j=1}^{N} \mathrm{e}^{-\frac{\left|x_{j}-y_{j}\right|^{2}}{4 t}}, x=\left(x_{1}, x_{2}, \cdots, x_{N}\right),
$$

the above calculation can be carried out successively for the variables $x_{i}, i=$ $1, \cdots, m$, yielding the desired formula.

This enables us to prove the following relationship between solutions of the linear heat equation in $C_{0}\left(\mathbb{R}^{N}\right)$ and $C_{0}(\Omega)$, in the sense of Definition 2.1.

Proposition 3.2. Let $\Psi:(0, \infty) \rightarrow C_{0}\left(\mathbb{R}^{N}\right)$ be a solution of the linear heat equation such that $T_{i} \Psi(t)=-\Psi(t)$ for all $1 \leq i \leq m$. It follows that $\Phi:(0, \infty) \rightarrow C_{0}(\Omega)$ defined by $\Phi(t)=\left.\Psi(t)\right|_{\bar{\Omega}}$ is a solution to the linear heat equation.

Let $\Phi:(0, \infty) \rightarrow C_{0}(\Omega)$ be a solution of the linear heat equation. It follows that $\Psi:(0, \infty) \rightarrow C_{0}\left(\mathbb{R}^{N}\right)$ is a solution of the linear heat equation, where $\Psi(t)=\tilde{\Phi}(t)$ is the unique extension of $\Phi(t)$ to $\mathbb{R}^{N}$ such that $T_{i} \Psi(t)=-\Psi(t)$ for all $1 \leq i \leq m$.

Proof. The first statement follows from (3.6), since

$$
\mathrm{e}^{t \Delta_{\Omega}} \Phi(s)=\mathrm{e}^{t \Delta_{\Omega}}\left(\left.\Psi(s)\right|_{\bar{\Omega}}\right)=\left.\left(\mathrm{e}^{t \Delta} \Psi(s)\right)\right|_{\bar{\Omega}}=\left.\Psi(t+s)\right|_{\bar{\Omega}}=\Phi(t+s) .
$$

The other statement is proved similarly, using the fact that $\widetilde{\mathrm{e}^{t \Delta_{\Omega} f}}=\mathrm{e}^{t \Delta} \tilde{f}, f \in$ $C_{0}(\Omega)$, which follows from Proposition 3.1. In fact, since $\Phi(t+s)=\mathrm{e}^{t \Delta_{\Omega}} \Phi(s)$, then

$$
\Psi(t+s)=\mathrm{e}^{t \widetilde{\Delta_{\Omega} \Phi(}(s)}=\mathrm{e}^{t \Delta} \tilde{\Phi}(s)=\mathrm{e}^{t \Delta} \Psi(s) .
$$

Let $\Psi:(0, \infty) \rightarrow C_{0}\left(\mathbb{R}^{N}\right)$ be a solution to the linear heat equation such that $T_{i} \Psi(t)=-\Psi(t)$ for all $1 \leq i \leq m$ and let $\Phi:(0, \infty) \rightarrow C_{0}(\Omega)$ be the solution of the linear heat equation on $\Omega$ defined by $\Phi(t)=\left.\Psi(t)\right|_{\Omega}$. Note that $\|\Psi(t)\|_{L^{\infty}\left(\mathbb{R}^{N}\right)}=$ $\|\Phi(t)\|_{L^{\infty}(\Omega)}$ for all $t>0$. The proof of the following proposition is now obvious.

Proposition 3.3. Let $\Psi:(0, \infty) \rightarrow C_{0}\left(\mathbb{R}^{N}\right)$ be a solution to the linear heat equation such that $T_{i} \Psi(t)=-\Psi(t)$ for all $1 \leq i \leq m$ and let $\Phi:(0, \infty) \rightarrow C_{0}(\Omega)$ be the solution of the linear heat equation on $\Omega$ defined by $\Phi(t)=\left.\Psi(t)\right|_{\bar{\Omega}}$, where $\Omega$ is the 
domain given by (3.3). Suppose $\Phi(t, x)>0$ for all $t>0$ and all $x \in \Omega$. For each $T>0$, let $X=X_{T, \Phi}$ be the space of functions given in Definition 2.2. Suppose $\alpha>0$ is such that condition (2.3) of Theorem 2.3 holds, and let $K>0, M>0$ and $T>0$ satisfy (2.4) and (2.5). Let $\varphi \in X_{T}$ such that $\|\varphi\| \| \leq K$ and let $u \in X_{T}$ be the unique solution with $\||u \|| \leq M$ of the integral equation

$$
u(t)=\varphi(t)+\int_{0}^{t} \mathrm{e}^{(t-\sigma) \Delta_{\Omega}}\left(|u(\sigma)|^{\alpha} u(\sigma)\right) d \sigma,
$$

given by Theorem 2.3 , Let $\tilde{u}:(0, T] \rightarrow C_{0}\left(\mathbb{R}^{N}\right)$ and $\tilde{\varphi}:(0, T] \rightarrow C_{0}\left(\mathbb{R}^{N}\right)$ be the extensions to $\mathbb{R}^{N}$ of $u$ and $\varphi$ such that $\tilde{u}(t)$ and $\tilde{\varphi}(t)$ all satisfy (3.5). It follows that

$$
\tilde{u}(t)=\tilde{\varphi}(t)+\int_{0}^{t} \mathrm{e}^{(t-\sigma) \Delta}\left(|\tilde{u}(\sigma)|^{\alpha} \tilde{u}(\sigma)\right) d \sigma,
$$

for all $0<t \leq T$. The integral in $(3.8)$ is absolutely convergent in $C_{0}\left(\mathbb{R}^{N}\right)$ and

$$
\int_{0}^{t}\left\|\mathrm{e}^{(t-\sigma) \Delta}\left(|\tilde{u}(\sigma)|^{\alpha} \tilde{u}(\sigma)\right)\right\|_{\infty} d \sigma \leq M^{\alpha+1}\|\Phi(t)\|_{\infty} \int_{0}^{t}\|\Phi(\sigma)\|_{\infty}^{\alpha} d \sigma .
$$

This last estimate follows from (2.10).

Proposition 3.4. Under the hypotheses of Proposition 3.3 , if, in addition, $\Phi(t)=$ $\Phi_{0}(t)$, given by (3.2), and $\alpha<2 /(N+m)$ so that (2.3) holds, it follows that

$$
\tilde{u}(t)-\tilde{\varphi}(t)=\int_{0}^{t} \mathrm{e}^{(t-\sigma) \Delta}\left(|\tilde{u}(\sigma)|^{\alpha} \tilde{u}(\sigma)\right) d \sigma \rightarrow 0
$$

in $\mathcal{S}^{\prime}\left(\mathbb{R}^{N}\right)$ as $t \rightarrow 0$.

For the proof of the last proposition, we need the following two lemmas.

Lemma 3.5. For $t>0$, we have

$$
\int_{\mathbb{R}^{N}} \Phi_{0}(t, x) x_{1} \cdots x_{m} d x=1 .
$$

Proof. This is a straightforward calculation since

$$
\Phi_{0}(t, x) x_{1} \cdots x_{m}=H_{t}(x)
$$

where

$$
H_{t}(x)=t^{-N / 2} H_{1}\left(\frac{x}{\sqrt{t}}\right), H_{1}(x)=(4 \pi)^{-\frac{N}{2}}(2)^{-m} \mathrm{e}^{-\frac{|x|^{2}}{4}}\left(\prod_{i=1}^{m} x_{i}\right)^{2}, t>0, x \in \mathbb{R}^{N},
$$

and $\int_{\mathbb{R}^{N}} H_{1}(x) d x=1$.

Lemma 3.6. Assume that $f \in C^{m}\left(\mathbb{R}^{N}\right)$ and $f$ is anti-symmetric with respect to the variables $x_{1}, \cdots, x_{m}$; that is, $f$ verifies (3.5). It follows that

$$
f(x)=\int_{0}^{x_{m}} \cdots \int_{0}^{x_{1}} \partial_{1} \cdots \partial_{m} f\left(t_{1}, \cdots, t_{m}, x_{m+1}, \cdots, x_{N}\right) d t_{1} \cdots d t_{m},
$$

for all $x \in \mathbb{R}^{N}$. In particular,

$$
|f(x)| \leq\left(\sup _{\mathbb{R}^{N}}\left|\partial_{1} \cdots \partial_{m} f\right|\right)\left|x_{1} x_{2} \cdots x_{m}\right|, \quad \forall x \in \mathbb{R}^{N} .
$$


Proof. To prove equality (3.10), we argue by induction on $m$. For $m=1$, the formula (3.10) is obvious. Assume that (3.10) is satisfied for $m-1$. Since $f$ is antisymmetric with respect to $x_{1}, \cdots, x_{m-1}$, then $\partial_{m} f$ is anti-symmetric with respect to $x_{1}, \cdots, x_{m-1}$, and then by the induction hypothesis,

$$
\begin{gathered}
\partial_{m} f\left(x_{1}, \cdots, x_{m-1}, t_{m}, x_{m+1}, \cdots, x_{N}\right) \\
=\int_{0}^{x_{m-1}} \cdots \int_{0}^{x_{1}} \partial_{1} \cdots \partial_{m-1} \partial_{m} f\left(t_{1}, t_{2}, \cdots, t_{m-1}, t_{m}, x_{m+1}, \cdots, x_{N}\right) d t_{1} d t_{2} \cdots d t_{m-1} .
\end{gathered}
$$

Integrating the last equality with respect to $t_{m}$ and using the anti-symmetry of $f$ with respect to $x_{m}$, we get (3.10) for $m$. (3.11) follows by (3.10) since $f$ is $C^{m}$.

Proof of Proposition 3.4. We begin by noting that if $f, g \in C_{0}(\Omega)$, with $\tilde{f}, \tilde{g}$ their extensions to $C_{0}\left(\mathbb{R}^{N}\right)$ verifying (3.5), and if $\tilde{g} \in L^{1}\left(\mathbb{R}^{N}\right)$, then

$$
\langle\tilde{f}, \tilde{g}\rangle_{\mathbb{R}^{N}}=2^{m}\langle f, g\rangle_{\Omega},
$$

where $\langle\tilde{f}, \tilde{g}\rangle_{\mathbb{R}^{N}}$ denotes $\int_{\mathbb{R}^{N}} \tilde{f}(x) \tilde{g}(x) d x$ and $\langle f, g\rangle_{\Omega}$ denotes $\int_{\Omega} f(x) g(x) d x$.

Let $\eta \in \mathcal{S}\left(\mathbb{R}^{N}\right)$. We need to show that

$$
\begin{aligned}
\lim _{t \rightarrow 0}\langle & \left\langle\int_{0}^{t} \mathrm{e}^{(t-\sigma) \Delta}\left(|\tilde{u}(\sigma)|^{\alpha} \tilde{u}(\sigma)\right) d \sigma, \eta\right\rangle_{\mathbb{R}^{N}} \\
& =\lim _{t \rightarrow 0} \int_{0}^{t}\left\langle\mathrm{e}^{(t-\sigma) \Delta}\left(|\tilde{u}(\sigma)|^{\alpha} \tilde{u}(\sigma)\right), \eta\right\rangle_{\mathbb{R}^{N}} d \sigma=0 .
\end{aligned}
$$

Since $\mathrm{e}^{(t-\sigma) \Delta}\left(|\tilde{u}(\sigma)|^{\alpha} \tilde{u}(\sigma)\right)$ satisfies (3.5) for every $0<\sigma<t$, it follows that if $\eta$ is symmetric in any one of the variables $x_{1}, \cdots, x_{m}$, i.e. if $T_{i} \eta=\eta$ for any one $i \in\{1,2, \cdots, m\}$, then

$$
\left\langle\mathrm{e}^{(t-\sigma) \Delta}\left(|\tilde{u}(\sigma)|^{\alpha} \tilde{u}(\sigma)\right), \eta\right\rangle_{\mathbb{R}^{N}}=\int_{\mathbb{R}^{N}}\left[\mathrm{e}^{(t-\sigma) \Delta}\left(|\tilde{u}(\sigma)|^{\alpha} \tilde{u}(\sigma)\right)\right](x) \eta(x) d x=0 .
$$

On the other hand, as is shown in the appendix, any function on $\mathbb{R}^{N}$ can be written explicitly as the sum of functions, one of which verifies (3.5), i.e. is anti-symmetric in all the variables $x_{1}, \ldots, x_{m}$, plus other functions, each of which is symmetric in at least one of the variables $x_{1}, \cdots, x_{m}$. This decomposition, which generalizes the fact that a function of one variable is the sum of an even and an odd function, preserves all the differentiability and decay properties of the original function; see (6.1). (Formula (6.1) is for the case $m=N$. The general case $1 \leq m \leq N$ is similar.) Thus, replacing $\eta$ by this decomposition, using the fact just noted that the pairing is zero for functions which are symmetric with respect to any one of the variables, we may suppose from now on that $\eta \in \mathcal{S}\left(\mathbb{R}^{N}\right)$ satisfies condition (3.5), i.e. is anti-symmetric with respect to all the variables $x_{1}, \cdots, x_{m}$. As such, it restricts to an element of $C_{0}(\Omega)$ still denoted by $\eta$. By the remark made at the start of the proof, it suffices to show that

$$
\begin{aligned}
\lim _{t \rightarrow 0} & \int_{0}^{t}\left\langle\mathrm{e}^{(t-\sigma) \Delta_{\Omega}}\left(|u(\sigma)|^{\alpha} u(\sigma)\right), \eta\right\rangle_{\Omega} d \sigma \\
& =\lim _{t \rightarrow 0} \int_{0}^{t} \int_{\Omega}\left[\mathrm{e}^{(t-\sigma) \Delta_{\Omega}}\left(|u(\sigma)|^{\alpha} u(\sigma)\right)\right](x) \eta(x) d x d \sigma=0 .
\end{aligned}
$$


As in the proof of Theorem 2.3, we see that, on $\Omega$, using $|u(\sigma)| \leq M \Phi_{0}(\sigma)$,

$$
\begin{aligned}
\left|\mathrm{e}^{(t-\sigma) \Delta_{\Omega}}\left(|u(\sigma)|^{\alpha} u(\sigma)\right)\right| & \leq \mathrm{e}^{(t-\sigma) \Delta_{\Omega}}(|u(\sigma)|)\|u(\sigma)\|_{\infty}^{\alpha} \\
& \leq \mathrm{e}^{(t-\sigma) \Delta_{\Omega}}\left(M \Phi_{0}(\sigma)\right) M^{\alpha}\left\|\Phi_{0}(\sigma)\right\|_{\infty}^{\alpha} \\
& =M^{\alpha+1} \Phi_{0}(t)\left\|\Phi_{0}(\sigma)\right\|_{\infty}^{\alpha},
\end{aligned}
$$

and so

$$
\left|\int_{0}^{t} \int_{\Omega}\left[\mathrm{e}^{(t-\sigma) \Delta_{\Omega}}\left(|u(\sigma)|^{\alpha} u(\sigma)\right)\right](x) \eta(x) d x d \sigma\right| \leq M^{\alpha+1}\left\langle\Phi_{0}(t),|\eta|\right\rangle_{\Omega} \int_{0}^{t}\left\|\Phi_{0}(\sigma)\right\|_{\infty}^{\alpha} d \sigma .
$$

Thus, to establish (3.13), it suffices by (3.14) to show that $\left\langle\Phi_{0}(t),|\eta|\right\rangle_{\Omega}$ is bounded as $t \rightarrow 0$.

Since $\eta \in \mathcal{S}\left(\mathbb{R}^{N}\right)$ and verifies (3.5), it follows from Lemma 3.6. specifically formula (3.11), that there exists $R>0$ such that

$$
|\eta(x)| \leq R\left|x_{1} x_{2} \cdots x_{m}\right|, \forall x \in \mathbb{R}^{N} .
$$

Thus,

$$
\begin{aligned}
\left|\left\langle\Phi_{0}(t),|\eta|\right\rangle_{\Omega}\right| & \leq R \int_{\Omega} \Phi_{0}(t, x) x_{1} x_{2} \cdots x_{m} d x \\
& =2^{-m} R \int_{\mathbb{R}^{N}} \Phi_{0}(t, x) x_{1} x_{2} \cdots x_{m} d x \\
& =2^{-m} R,
\end{aligned}
$$

where we have used Lemma 3.5. This finishes the proof of the proposition.

Proof of Theorem 1.1. Consider $\Phi_{0}(t)$, given by (3.2) and (3.4). Since

$$
\Phi_{0}(t, x)=t^{-(N+m) / 2} \Phi_{0}(1, x / \sqrt{t}),
$$

it is clear that

$$
\left\|\Phi_{0}(t)\right\|_{\infty}=\left\|\Phi_{0}(1)\right\|_{\infty} t^{-(N+m) / 2} .
$$

Thus, if we consider $\Phi_{0}(t)$ as a solution of the linear heat equation in $C_{0}(\Omega)$, we may apply Theorem 2.3 for $\alpha<2 /(N+m)$. Let $K>0$. Choose $M>0$ and $T>0$ so that (2.4) and (2.5) are satisfied. (If $K<0$, we replace $K$ by $|K|$ in (2.4) and (2.5).) We set $\varphi(t)=K \Phi_{0}(t)$. Theorem 2.3 gives a local solution to the integral equation (3.7) in $C_{0}(\Omega)$ and therefore by Proposition 3.3 a solution of (3.8) in $C_{0}\left(\mathbb{R}^{N}\right)$. Since $\varphi(t)>0$ on $\Omega$, it is clear that $u(t)>0$ on $\Omega$. (If $K<0$, then $u(t)<0$ on $\Omega$.) By abuse of notation, we write $u$ and $\varphi$ instead of $\tilde{u}$ and $\tilde{\varphi}$, and we note that in $\mathbb{R}^{N}, \varphi(t)=\mathrm{e}^{t \Delta} u_{0}$, where $u_{0}=K(-1)^{m} \partial_{1} \partial_{2} \cdots \partial_{m} \delta$. We thus obtain a solution to the integral equation (1.1) in $\mathbb{R}^{N}$ with $u_{0}=K(-1)^{m} \partial_{1} \partial_{2} \cdots \partial_{m} \delta$. The last statement follows by Proposition 3.4 .

\section{An extension of Dickstein's "SMall lambda" Blow Up Result}

We continue with the notation of Section 3 and assume that $\alpha<2 /(N+m)$. In particular, $\Phi_{0}(t)$ is given by (3.2) and (3.4), and $\Omega$ is given by (3.3). Before proving Theorem 1.2, we need the following preliminary result concerning the hypotheses of that theorem. 
Proposition 4.1. Let $f \in C_{0}\left(\mathbb{R}^{N}\right)$ be anti-symmetric with respect to the variables $x_{1}, \cdots, x_{m}$, i.e. such that $f$ satisfies condition (3.5), and suppose there exist $t_{0}>0$ and $K>0$ such that

$$
|f(x)| \leq K\left|\Phi_{0}\left(t_{0}, x\right)\right|
$$

for all $x \in \mathbb{R}^{N}$. Let $g: \mathbb{R}^{N} \rightarrow \mathbb{R}$ be given by

$$
\begin{aligned}
& g\left(x_{1}, x_{2}, \cdots, x_{N}\right) \\
& \quad=(-1)^{m} \int_{-\infty}^{x_{1}} \cdots \int_{-\infty}^{x_{m}} f\left(y_{1}, y_{2}, \cdots, y_{m}, x_{m+1}, \cdots, x_{N}\right) d y_{1} d y_{2} \cdots d y_{m} .
\end{aligned}
$$

It follows that $g \in C_{0}\left(\mathbb{R}^{N}\right)$ and

$$
f=(-1)^{m} \partial_{1} \partial_{2} \cdots \partial_{m} g
$$

Furthermore, $g$ decays exponentially as $|x| \rightarrow \infty$, so (in particular) $g \in L^{1}\left(\mathbb{R}^{N}\right.$ ) and

$$
\int_{\mathbb{R}^{N}} g=\int_{\mathbb{R}^{N}} x_{1} x_{2} \cdots x_{m} f(x) d x_{1} d x_{2} \cdots d x_{N}
$$

If we set

$$
f_{\tau}(x)=\tau^{-(N+m) / 2} f\left(\frac{x}{\sqrt{\tau}}\right),
$$

then, for any given $t>0$,

$$
\mathrm{e}^{t \Delta} f_{\tau} \rightarrow \mathrm{e}^{t \Delta} K_{0}(-1)^{m} \partial_{1} \partial_{2} \cdots \partial_{m} \delta=K_{0} \Phi_{0}(t)
$$

in $\mathcal{S}^{\prime}\left(\mathbb{R}^{N}\right)$ as $\tau \rightarrow 0$, where

$$
K_{0}=\int_{\mathbb{R}^{N}} g .
$$

Finally, it must be that $\left|K_{0}\right| \leq K$.

Proof. The fact that $g \in C_{0}\left(\mathbb{R}^{N}\right)$ is an immediate consequence of (4.1) and the anti-symmetry of $f$ in the first $m$ variables. The decay estimate follows easily since $\left|\Phi_{0}\left(t_{0}, x\right)\right| \leq \mathrm{e}^{-\left(\left|x_{1}\right|+\cdots+\left|x_{N}\right|\right)}$ for sufficiently large $|x|$ and the anti-symmetry of $f$. To show (4.4), we use a calculation inspired by the proof of Corollary 1 in [8]. We note that $g$ is symmetric with respect to $x_{1}, \cdots, x_{m}$ so that $\int_{\mathbb{R}^{N}} g$ is given by

$(-1)^{m} 2^{m} \int_{\mathbb{R}^{N-m}} \int_{-\infty}^{0} \cdots \int_{-\infty}^{0} \int_{-\infty}^{x_{m}} \cdots \int_{-\infty}^{x_{1}} f\left(y_{1}, y_{2}, \cdots, y_{m}, x_{m+1}, \cdots, x_{N}\right) d y d x$,

where $d y=d y_{1} \cdots d y_{m}$ and $d x=d x_{1} \cdots d x_{N}$. Interchanging the order of integration between each $x_{j}$ and $y_{j}$, for $1 \leq j \leq m$, we see that $\int_{\mathbb{R}^{N}} g$ is given by

$$
\begin{aligned}
& (-1)^{m} 2^{m} \int_{\mathbb{R}^{N-m}} \int_{-\infty}^{0} \cdots \int_{-\infty}^{0} \int_{y_{m}}^{0} \cdots \int_{y_{1}}^{0} f\left(y_{1}, y_{2}, \cdots, y_{m}, x_{m+1}, \cdots, x_{N}\right) \\
& \times d x_{1} d x_{2} \cdots d x_{m} d y d x^{\prime}
\end{aligned}
$$

where $d x^{\prime}=d x_{m+1} \cdots d x_{N}$. This equals

$$
2^{m} \int_{\mathbb{R}^{N-m}} \int_{-\infty}^{0} \cdots \int_{-\infty}^{0} y_{1} \cdots y_{m} f\left(y_{1}, y_{2}, \cdots, y_{m}, x_{m+1}, \cdots, x_{N}\right) d y d x^{\prime}
$$

which is precisely the right hand side of (4.4). 
If we now set

then clearly

$$
g^{\tau}(x)=\tau^{-N / 2} g\left(\frac{x}{\sqrt{\tau}}\right),
$$

$$
g^{\tau} \rightarrow K_{0} \delta
$$

in $\mathcal{S}^{\prime}\left(\mathbb{R}^{N}\right)$ as $\tau \rightarrow 0$, where $K_{0}$ is given by (4.7). Since $f_{\tau}=(-1)^{m} \partial_{1} \partial_{2} \cdots \partial_{m} g^{\tau}$, it follows that

$$
f_{\tau} \rightarrow K_{0}(-1)^{m} \partial_{1} \partial_{2} \cdots \partial_{m} \delta
$$

in $\mathcal{S}^{\prime}\left(\mathbb{R}^{N}\right)$, as $\tau \rightarrow 0$. This implies (4.6). Finally, we prove that $\left|K_{0}\right| \leq K$. Indeed, since $f$ is anti-symmetric with respect to the variables $x_{1}, \cdots, x_{m}$ it follows that $g$ is symmetric with respect to $x_{1}, \cdots, x_{m}$. Thus

$$
K_{0}=2^{m} \int_{-\infty}^{0} \cdots \int_{-\infty}^{0} \int_{\mathbb{R}^{N-m}} g\left(x_{1}, x_{2}, \cdots, x_{m}, x^{\prime}\right) d x, x=\left(x_{1}, x_{2} \cdots, x_{m}, x^{\prime}\right) .
$$

Using the fact that

$$
\left|\Phi_{0}\left(t_{0}, x\right)\right|=\partial_{1} \partial_{2} \cdots \partial_{m} G_{t_{0}}(x), t_{0}>0, x \in \mathbb{R}^{N}, x_{i}<0,
$$

for $i \leq m$, it follows that

$$
\begin{aligned}
\left|K_{0}\right| & \leq 2^{m} \int_{-\infty}^{0} \cdots \int_{-\infty}^{0} \int_{\mathbb{R}^{N-m}}\left|g\left(x_{1}, x_{2}, \cdots x_{m}, x^{\prime}\right)\right| d x_{1} d x_{2} \cdots d x_{m} d x^{\prime} \\
& \leq 2^{m} K \int_{-\infty}^{0} \cdots \int_{-\infty}^{0} \int_{\mathbb{R}^{N-m}}\left[\int_{-\infty}^{x_{1}} \cdots \int_{-\infty}^{x_{m}}\left|\Phi_{0}\left(t_{0}, y_{1}, y_{2}, \ldots, y_{m}, x^{\prime}\right)\right|\right. \\
& \left.\left.=2^{m} K \int_{\mathbb{R}^{N-m}} \int_{-\infty}^{m} \cdots \int_{-\infty}^{m} d y_{i}\right)\right] d x \\
& \leq K \int_{\mathbb{R}^{N-m}} 2^{m} \int_{-\infty}^{0} \cdots \int_{-\infty}^{x_{1}} \cdots \int_{-\infty}^{x_{m}} \partial_{1} \cdots \partial_{m} G_{t_{0}}\left(y_{1}, \cdots, y_{m}, x^{\prime}\right) \\
& \left.\leq K \int_{\mathbb{R}^{N-m}} \int_{\mathbb{R}^{m}} G_{t_{0}}\left(\prod_{1}, x_{2}, \cdots, x_{2}, \cdots, x_{m}\right)\right] d x \\
&
\end{aligned}
$$

Remark 4.2. A class of examples of functions $f$ as in the assumptions of Proposition 4.1 is not hard to construct. Let $f \in C^{m}\left(\mathbb{R}^{N}\right)$ with compact support and $f$ be anti-symmetric with respect to the variables $x_{1}, \cdots, x_{m}$. We claim that for any $t_{0}>0$, there exists $K>0$ such that (4.1) holds on $\mathbb{R}^{N}$. To see this, we note that (3.4) implies that on any bounded set in $\mathbb{R}^{N}$, there exists $C_{1}>0$ such that $\left|\Phi_{0}\left(t_{0}, x\right)\right| \geq C_{1}\left|x_{1} x_{2} \cdots x_{m}\right|$. Thus, the claim follows from Lemma 3.6. We may replace the condition on the compactness of the support of $f$ by assuming that $f$ has an appropriate exponential decaying with $\sup _{\mathbb{R}^{N}}\left|\partial_{1} \partial_{2} \cdots \partial_{m} f\right|<\infty$. 
Proof of Theorem 1.2. Let $f \in C_{0}\left(\mathbb{R}^{N}\right)$ be as in the hypotheses of Theorem 1.2. i.e. as in the hypotheses of Proposition 4.1 with $K_{0} \neq 0$. We assume for notational simplicity that $t_{0}=1$. (The general case can be treated by the same arguments, but with an additional factor of $t_{0}$ appearing in many formulas.) Also, we treat the case $K_{0}>0$. We consider the maximal solution in $C_{0}\left(\mathbb{R}^{N}\right)$ of (1.1) with initial value $u_{0}=\lambda f$, for $\lambda>0$. We wish to show that this solution blows up in finite time if $\lambda>0$ is sufficiently small. By the standard invariance properties of solutions to (1.4) and (1.1), this is equivalent to showing that the maximal solution with initial value

$$
u_{0, \tau}(x)=\lambda \tau^{-1 / \alpha} f\left(\frac{x}{\sqrt{\tau}}\right)
$$

blows up in finite time for any $\tau>0$. For a given $\lambda>0$ we let $\tau=\tau(\lambda)$ be such that

$$
\lambda=\tau^{-\frac{N+m}{2}+\frac{1}{\alpha}} .
$$

With this definition of $\tau$, it follows that $u_{0, \tau}=f_{\tau}$, given by (4.5), and that as $\lambda \rightarrow 0$, so does $\tau \rightarrow 0$ (since $\alpha<2 /(N+m)$ ). It thus suffices to show that the maximal solution of (1.1) with initial value $f_{\tau}$ blows up in finite time, for $\tau>0$ sufficiently small. The basic idea is to prove this using the fact that $f_{\tau} \rightarrow K_{0}(-1)^{m} \partial_{1} \partial_{2} \cdots \partial_{m} \delta$ in $\mathcal{S}^{\prime}\left(\mathbb{R}^{N}\right)$, as $\tau \rightarrow 0$, and applying the machinery developed thus far in this paper. To do this, we start with the initial value $K_{0}(-1)^{m} \partial_{1} \partial_{2} \cdots \partial_{m} \delta$.

We let $u:(0, T] \rightarrow C_{0}\left(\mathbb{R}^{N}\right)$ be the local solution of the integral equation (1.1), constructed using Theorem 2.3, with $\Phi(t)=\Phi_{0}(t)$, and Proposition 3.3, with initial value

$$
u_{0}=K_{0}(-1)^{m} \partial_{1} \partial_{2} \cdots \partial_{m} \delta .
$$

In other words, we apply Theorem 2.3 with $\varphi(t)=K_{0} \Phi_{0}(t)$ in $C_{0}(\Omega)$. However, in the application of Theorem 2.3. we use the value of $K$ above, rather than $K_{0}$. This is possible since $\left.|||\varphi(t)|\right|_{X}=\left|K_{0}\right| \leq K$. The constants $K, M$ and $T$ satisfy (2.4) and (2.5) and $|u(t)| \leq M\left|\Phi_{0}(t)\right|$ for all $t \in(0, T]$. Thus, by Proposition 3.3. we have

$$
u(t)=\mathrm{e}^{t \Delta} u_{0}+\int_{0}^{t} \mathrm{e}^{(t-\sigma) \Delta}\left(|u(\sigma)|^{\alpha} u(\sigma)\right) d \sigma .
$$

We suppress the "tilde" and use the same notation for a function in $C_{0}(\Omega)$ and its anti-symmetric extension to a function in $C_{0}\left(\mathbb{R}^{N}\right)$ so that $u(t)$, as an element of $C_{0}\left(\mathbb{R}^{N}\right)$, satisfies condition (3.5) and restricts to an element of $C_{0}(\Omega)$. This is essentially the same solution that was described in Theorem 1.1. Since $K_{0}>0$, it follows that $u(t)>0$ on $\Omega$.

Next, for each $\tau>0$, we let $v_{\tau}:\left[0, T_{\tau}\right) \rightarrow C_{0}\left(\mathbb{R}^{N}\right)$ be the maximal solution of the integral equation (1.1) with initial value $f_{\tau}$. We observe that $T<T_{\tau}$ since $v_{\tau}$ can also be constructed using Theorem 2.3 and Proposition 3.3 with the same values of $K, M$ and $T$ as just above. Indeed, $\left|f_{\tau}(x)\right| \leq K \Phi_{0}(\tau, x)$, and so we set $\Phi_{\tau}(t)=$ $\Phi_{0}(t+\tau)$ and use the space $X_{T, \Phi_{\tau}}$ in Theorem 2.3. Since $\left\|\Phi_{\tau}(t)\right\|_{\infty} \leq\left\|\Phi_{0}(t)\right\|_{\infty}$, the same values of $K, M$ and $T$ satisfy (2.4) and (2.5) with $\Phi(t)=\Phi_{\tau}(t)$ as well as $\Phi(t)=\Phi_{0}(t)$. Also, we use $\varphi_{\tau}(t)=\mathrm{e}^{t \Delta} f_{\tau}$, and so (on $\left.\Omega\right)|\varphi(t)| \leq K \mathrm{e}^{t \Delta_{\Omega}} \Phi_{0}(\tau)=$ $K \Phi_{0}(t+\tau)=K \Phi_{\tau}(t)$. This means that $\left\|\left|\varphi_{\tau}\right|\right\|_{X_{T, \Phi_{\tau}}} \leq K$. We therefore have

$$
v_{\tau}(t)=\mathrm{e}^{t \Delta} f_{\tau}+\int_{0}^{t} \mathrm{e}^{(t-\sigma) \Delta}\left(\left|v_{\tau}(\sigma)\right|^{\alpha} v_{\tau}(\sigma)\right) d \sigma
$$


and that $\left.||\left|v_{\tau}\right|\right|_{X_{T, \Phi_{\tau}}} \leq M$. In particular, $\left|v_{\tau}(t, x)\right| \leq M \Phi_{0}(t+\tau, x)$ for all $0<t \leq T$ and $x \in \Omega$, and since $v_{\tau}$ satisfies (3.5),,$\left|v_{\tau}(t, x)\right| \leq M\left|\Phi_{0}(t+\tau, x)\right|$ for all $0<t \leq T$ and $x \in \mathbb{R}^{N}$. [For the reader's information, if we had not chosen $t_{0}=1$, then in many of these formulas it would have been necessary to replace $\tau$ by $\tau t_{0}$.]

We wish to show that for each $0<t \leq T, v_{\tau}(t) \rightarrow u(t)$ in $C_{0}\left(\mathbb{R}^{N}\right)$ as $\tau \rightarrow 0$. To accomplish this, we will show, with the help of the dominated convergence theorem, that, passing to a subsequence, the $v_{\tau}$ converge to a solution of (4.8) in $X_{T, \Phi_{0}}$. By the uniqueness part of Theorem 2.3 that limit must be the solution $u$ above. Also, by uniqueness, the limit exists as $\tau \rightarrow 0$, not just along subsequences.

We fix $0<t \leq T$ and let $h_{\tau}:[0, t] \rightarrow C_{0}\left(\mathbb{R}^{N}\right)$ and $H_{\tau}:[0, t] \rightarrow C_{0}\left(\mathbb{R}^{N}\right)$ be given by

$$
h_{\tau}(\sigma)=\mathrm{e}^{(t-\sigma) \Delta}\left(\left|v_{\tau}(\sigma)\right|^{\alpha} v_{\tau}(\sigma)\right)
$$

and

$$
H_{\tau}(\sigma)=M^{\alpha+1}\left|\Phi_{0}(t+\tau)\right|\left\|\Phi_{0}(\sigma+\tau)\right\|_{\infty}^{\alpha} .
$$

It is clear that $H_{\tau} \rightarrow H$ in $L^{1}\left((0, t) ; C_{0}\left(\mathbb{R}^{N}\right)\right)$ where

$$
H(\sigma)=M^{\alpha+1}\left|\Phi_{0}(t)\right|\left\|\Phi_{0}(\sigma)\right\|_{\infty}^{\alpha} .
$$

Moreover, it follows from the construction of the $v_{\tau}$ that

$$
h_{\tau}(\sigma) \leq H_{\tau}(\sigma)
$$

for all $0 \leq \sigma \leq t$. To see this, it suffices to show the inequality on $\Omega$, in which case we have

$$
\begin{aligned}
h_{\tau}(\sigma) & \leq\left(\mathrm{e}^{(t-\sigma) \Delta_{\Omega}}\left|v_{\tau}(\sigma)\right|\right)\left\|v_{\tau}(\sigma)\right\|_{\infty}^{\alpha} \\
& \leq M^{\alpha+1}\left(\mathrm{e}^{(t-\sigma) \Delta_{\Omega}} \Phi_{0}(\sigma+\tau)\right)\left\|\Phi_{0}(\sigma+\tau)\right\|_{\infty}^{\alpha} \\
& \leq M^{\alpha+1} \Phi_{0}(t+\tau)\left\|\Phi_{0}(\sigma+\tau)\right\|_{\infty}^{\alpha} \\
& =H_{\tau}(\sigma) .
\end{aligned}
$$

Next we fix $0<\sigma_{0}<t$. Since $\left|v_{\tau}\left(\sigma_{0}\right)\right| \leq M\left|\Phi_{0}\left(\sigma_{0}+\tau\right)\right|$, it follows that for all $0<\tau<1$

$$
\left|v_{\tau}\left(\sigma_{0}\right)\right| \leq \Theta
$$

where

$$
\Theta=M \sup _{0 \leq \tau \leq 1}\left|\Phi_{0}\left(\sigma_{0}+\tau\right)\right| \in C_{0}\left(\mathbb{R}^{N}\right) \cap L^{1}\left(\mathbb{R}^{N}\right) .
$$

By parabolic regularity, applied to the $v_{\tau}\left(\sigma_{0}\right), 0<\tau<1$, as initial values of the nonlinear heat equation (1.4), it follows that the $\partial_{t} v_{\tau}(\sigma)$ and $\nabla v_{\tau}(\sigma)$ are uniformly bounded in $C_{0}\left(\mathbb{R}^{N}\right)$ on any interval $\left[\sigma_{1}, t\right]$ with $\sigma_{0}<\sigma_{1}<t$. By the Arzela-Ascoli theorem and a standard diagonalization procedure, there exists a sequence $\tau_{n} \rightarrow 0$ and a function $w \in C\left((0, t) \times \mathbb{R}^{N}\right)$ such that

$$
\lim _{n \rightarrow \infty} v_{\tau_{n}}(\sigma, x)=w(\sigma, x),
$$

where the limit is uniform for $\sigma$ in a closed subinterval of $(0, t)$ and $x$ in any bounded set of $\mathbb{R}^{N}$. Since $\left|v_{\tau}(\sigma, x)\right| \leq M\left|\Phi_{0}(\sigma+\tau, x)\right|$ for all $0<\sigma<t$ and $x \in \mathbb{R}^{N}$, it follows that $|w(\sigma, x)| \leq M\left|\Phi_{0}(\sigma, x)\right|$ and that the above limit takes place in $C_{0}\left(\mathbb{R}^{N}\right)$ for each $0<\sigma<t$.

If we denote $w(\sigma)=w(\sigma, \cdot)$, it is clear that

$$
h_{\tau_{n}}(\sigma) \rightarrow \mathrm{e}^{(t-\sigma) \Delta}\left(|w(\sigma)|^{\alpha} w(\sigma)\right)
$$


in $C\left((0, t) ; C_{0}\left(\mathbb{R}^{N}\right)\right.$, as $n \rightarrow \infty$. By the dominated convergence theorem and formulas (4.6) and (4.9), it now follows that

$$
w(t)=K_{0} \Phi_{0}(t)+\int_{0}^{t} \mathrm{e}^{(t-\sigma) \Delta}\left(|w(\sigma)|^{\alpha} w(\sigma)\right) d \sigma .
$$

As an element of $C_{0}\left(\mathbb{R}^{N}\right), w(t)$ satisfies condition (3.5), since it is the limit of functions satisfying (3.5), and so restricts to an element of $C_{0}(\Omega)$. Thus, we may consider (4.10) as an integral equation with values in $C_{0}(\Omega), w \in X_{T, \Phi_{0}}$ and $\||w|\|_{X_{T, \Phi_{0}}} \leq M$. By the uniqueness part of Theorem 2.3, it follows that $w=u$ as elements of $w \in X_{T, \Phi_{0}}$ and therefore as functions $(0, T] \rightarrow C_{0}\left(\mathbb{R}^{N}\right)$. It now follows by standard arguments that

$$
v_{\tau} \rightarrow u
$$

in $C\left((0, T) ; C_{0}\left(\mathbb{R}^{N}\right)\right.$ as $\tau \rightarrow 0$.

Since $\alpha<2 /(N+m)$, and since $u(t)>0$ on $\Omega$, it follows from the Fujita-type results on $\Omega([16,13,1,15])$ that the solution $u$, extended to its maximal solution $u:\left(0, T_{\max }\right) \rightarrow C_{0}\left(\mathbb{R}^{N}\right)$, blows up in finite time. We complete the proof, as done by Dickstein [4, and later Ghoul [7, using the fact that in the Sobolev subcritical case all blow up is type I (see Giga, Matsui, and Sasayama [9, 10]) and that the set of initial values giving rise to type I blow up is open in $C_{0}\left(\mathbb{R}^{N}\right)$ (see Fermanian Kammerer, Merle, and Zaag [5]). Using $v_{\tau}\left(t_{0}\right)$ and $u\left(t_{0}\right)$ as initial values, for some fixed $0<t_{0}<T$, we deduce that $v_{\tau}$ blows up in finite time for $\tau>0$ sufficiently small. This completes the proof.

Remark 4.3. The condition $\alpha<2 /(N+m)$ in Theorem 1.2 is optimal. If $\alpha>$ $2 /(N+m)$ and if $f$ is anti-symmetric in $x_{1}, x_{2}, \cdots, x_{m}$, satisfying (1.7) for some $t_{0}>0$, then the solution of the integral equation (1.1) with initial value $u_{0}=\lambda f$ is global for $\lambda>0$ sufficiently small. To see this we apply Theorem 2.3 with $\Phi=\Phi_{0}\left(t_{0}+\cdot\right)$. Thus, (2.3) is verified with $A=\infty$ since $\alpha>2 /(N+m)$. Let $K>0$ and $M>0$ be such that (2.4)-(2.5) are satisfied with $T=\infty$. (See Remark 2.6.) Let $\varphi=e^{t \Delta} u_{0}$. Then $|\varphi(t)| \leq K \Phi(t), \forall t \geq 0$ and $\lambda>0$ sufficiently small. That is, $\varphi \in X_{\infty}$ and $|\|\varphi\|| \leq K$. Then by Theorem 2.3 the solution of (1.1) with initial data $u_{0}$ is global. Of course, the case $m=0$ is just the $\alpha>2 / N$ part of Fujita's original result.

\section{Global existence: Proof of Theorem 1.3}

In this section, we continue with the notation that $\mathrm{e}^{t \Delta}$ denotes the heat semigroup on $\mathbb{R}^{N}$, $\mathrm{e}^{t \Delta} f=G_{t} \star f$, where $G_{t}$ is the Gaussian function given by (1.3). Recall that if $u_{0} \in C_{0}\left(\mathbb{R}^{N}\right)$, then there exists a unique continuous solution $u$ : $\left[0, T_{\max }\left(u_{0}\right)\right) \rightarrow C_{0}\left(\mathbb{R}^{N}\right)$ of (1.1) with $u(0)=u_{0}$. This solution is classical for $t>0$, i.e. $u \in C^{1,2}\left(\left(0, T_{\max }\right) \times \mathbb{R}^{N}\right)$.

Fix $\rho>0$, and let $u_{0} \in C_{0}\left(\mathbb{R}^{N}\right)$ be such that $\left|u_{0}\right| \leq K G_{\rho}$ for some $K>0$. Then the solution $u$ of (1.1) can also be constructed, at least on some smaller time interval, using Theorem 2.3. For this, we let $\Phi(t)=\mathrm{e}^{t \Delta} G_{\rho}=G_{t+\rho}$ and $\varphi(t)=\mathrm{e}^{t \Delta} u_{0}$. We clearly have that $|\varphi(t)| \leq K \Phi(t)$, i.e. $\||\varphi|\| X_{X_{T}} \leq K$ for all $T>0$. It follows that if $T>0$ and $M>0$ satisfy (2.4) and (2.5), then the solution of (1.1) satisfies $|u(t)| \leq M G_{t+\rho}$ for all $t \in[0, T]$. In this case, it turns out that the first and second order spatial derivatives of $u(t)$ are likewise bounded by Gaussian functions for $t>0$. 
Proposition 5.1. Let $\alpha>0, \rho>0$, and let $u_{0} \in C_{0}\left(\mathbb{R}^{N}\right)$ be such that $\left|u_{0}\right| \leq K G_{\rho}$ for some $K>0$. Set $\Phi(t)=\mathrm{e}^{t \Delta} G_{\rho}=G_{t+\rho}$, and suppose that $T>0$ and $M>0$ satisfy (2.4) and (2.5). Let $u \in X_{T}$ be the resulting solution of (1.1), i.e. (2.6) with $\varphi(t)=\mathrm{e}^{t \Delta} u_{0}$. It follows that for any $\epsilon>0$ there exists $C>0$, depending only on the various parameters: $K, M, T, \alpha, \rho$, as well as $\epsilon$, such that

$$
\begin{aligned}
\left|\partial_{k} u(t)\right| & \leq \frac{C}{\sqrt{t}} G_{(1+\epsilon)(t+\rho)}, \\
\left|\partial_{j k} u(t)\right| & \leq \frac{C}{t} G_{(1+\epsilon)(t+\rho)},
\end{aligned}
$$

for all $t \in(0, T]$ and all indices $1 \leq j, k \leq N$.

Proof. As noted just above the statement of the proposition, we have $|u(t)| \leq$ $M G_{t+\rho}$ for all $t \in[0, T]$. Further, we note that for any index $1 \leq k \leq N$,

$$
\partial_{k} G_{t}(x)=\frac{1}{2 \sqrt{t}} \frac{1}{(4 \pi t)^{N / 2}} \frac{\left(-x_{k}\right)}{\sqrt{t}} \mathrm{e}^{-\frac{|x|^{2}}{4 t}} .
$$

On the other hand, for any $\epsilon>0$, there exists $C_{\epsilon}$ such that

$$
\left|y_{k}\right| \mathrm{e}^{-\frac{|y|^{2}}{4}} \leq C_{\epsilon} \mathrm{e}^{-\frac{|y|^{2}}{4(1+\epsilon)}}
$$

from which we see that

$$
\left|\partial_{k} G_{t}\right| \leq \frac{C_{\epsilon}}{\sqrt{t}} G_{(1+\epsilon) t}
$$

where the value of $C_{\epsilon}$ may vary from line to line. Also, we note that

$$
\left(G_{t}\right)^{\beta}=\frac{1}{\beta^{N / 2}(4 \pi t)^{N(\beta-1) / 2}} G_{t / \beta}=\beta^{-N / 2}\left\|G_{t}\right\|_{\infty}^{\beta-1} G_{t / \beta} .
$$

For any index $1 \leq k \leq N$, we have

$$
\partial_{k} u(t)=\left(\partial_{k} G_{t}\right) \star u_{0}+\int_{0}^{t}\left(\partial_{k} G_{t-\sigma}\right) \star\left(|u(\sigma)|^{\alpha} u(\sigma)\right) d \sigma .
$$

Therefore, for any $0<\beta<\alpha+1$, we have

$$
\begin{aligned}
\left|\partial_{k} u(t)\right| \leq & \frac{C_{\epsilon}}{\sqrt{t}} G_{(1+\epsilon) t} \star K G_{\rho} \\
+ & M^{\alpha+1} C_{\epsilon} \int_{0}^{t}(t-\sigma)^{-1 / 2} G_{(1+\epsilon)(t-\sigma)} \star\left(G_{\sigma+\rho}\right)^{\alpha+1} d \sigma \leq \frac{K C_{\epsilon}}{\sqrt{t}} G_{(1+\epsilon) t+\rho} \\
& +M^{\alpha+1} C_{\epsilon} \int_{0}^{t}(t-\sigma)^{-1 / 2} G_{(1+\epsilon)(t-\sigma)} \star\left(G_{\sigma+\rho}\right)^{\beta}\left\|G_{\sigma+\rho}\right\|_{\infty}^{\alpha+1-\beta} d \sigma \\
& =\frac{K C_{\epsilon}}{\sqrt{t}} G_{(1+\epsilon) t+\rho} \\
+ & M^{\alpha+1} C_{\epsilon} \beta^{-N / 2} \\
& \times \int_{0}^{t}(t-\sigma)^{-1 / 2} G_{(1+\epsilon)(t-\sigma)} \star\left(G_{(\sigma+\rho) / \beta}\right)\left\|G_{\sigma+\rho}\right\|_{\infty}^{\beta-1}\left\|G_{\sigma+\rho}\right\|_{\infty}^{\alpha+1-\beta} d \sigma .
\end{aligned}
$$

We now choose $\beta=1 /(1+\epsilon)$ so that

$$
G_{(1+\epsilon)(t-\sigma)} \star G_{(\sigma+\rho) / \beta}=G_{(1+\epsilon)(t-\sigma)} \star G_{(1+\epsilon)(\sigma+\rho)}=G_{(1+\epsilon)(t+\rho)} .
$$


Inserting this into the previous calculation, we obtain (adjusting the value of $C_{\epsilon}$ as necessary)

$$
\begin{aligned}
\left|\partial_{k} u(t)\right| & \leq \frac{K C_{\epsilon}}{\sqrt{t}} G_{(1+\epsilon) t+\rho}+M^{\alpha+1} C_{\epsilon}\left\|G_{\rho}\right\|_{\infty}^{\alpha} G_{(1+\epsilon)(t+\rho)} \int_{0}^{t}(t-\sigma)^{-1 / 2} d \sigma \\
& =\frac{K C_{\epsilon}}{\sqrt{t}} G_{(1+\epsilon) t+\rho}+\sqrt{t} M^{\alpha+1} C_{\epsilon}\left\|G_{\rho}\right\|_{\infty}^{\alpha} G_{(1+\epsilon)(t+\rho)} .
\end{aligned}
$$

This yields

$$
\left|\partial_{k} u(t)\right| \leq \frac{C}{\sqrt{t}} G_{(1+\epsilon)(t+\rho)},
$$

where the constant $C$ now depends (only) on $K, M, T, \rho$ and $\epsilon$.

We now note that for any indices $1 \leq j, k \leq N$,

$$
\partial_{j k} G_{t}(x)=\frac{1}{t}\left(-\frac{\varepsilon_{j k}}{2}+\frac{x_{j} x_{k}}{4 t}\right) \frac{1}{(4 \pi t)^{N / 2}} \mathrm{e}^{-\frac{|x|^{2}}{4 t}},
$$

where $\varepsilon_{k k}=1$ and $\varepsilon_{j k}=0$ if $j \neq k$. By similar calculations as above, we see that

$$
\left|\partial_{j k} G_{t}\right| \leq \frac{C_{\epsilon}}{t} G_{(1+\epsilon) t} .
$$

For any two indices $1 \leq j, k \leq N$, we write

$$
\begin{aligned}
\partial_{j k} u(t) & =\left(\partial_{j k} G_{t}\right) \star u_{0}+\int_{0}^{t}\left(\partial_{j} G_{t-\sigma}\right) \star \partial_{k}\left(|u(\sigma)|^{\alpha} u(\sigma)\right) d \sigma \\
& =\left(\partial_{j k} G_{t}\right) \star u_{0}+(\alpha+1) \int_{0}^{t}\left(\partial_{j} G_{t-\sigma}\right) \star\left(\left(\partial_{k} u(\sigma)\right)|u(\sigma)|^{\alpha}\right) d \sigma .
\end{aligned}
$$

Using the estimates already obtained and denoting all the constants by the same letter $C$, we see that

$$
\begin{aligned}
\left|\partial_{j k} u(t)\right| & \leq \frac{C}{t} G_{(1+\epsilon) t} \star G_{\rho}+C \int_{0}^{t} \frac{1}{\sqrt{t-\sigma}} \frac{1}{\sqrt{\sigma}} G_{(1+\epsilon)(t-\sigma)} \star G_{(1+\epsilon)(\sigma+\rho)} d \sigma \\
& \leq \frac{C}{t} G_{(1+\epsilon)(t+\rho)} .
\end{aligned}
$$

This concludes the proof.

Lemma 5.2. Assume that $f \in C^{m}\left(\mathbb{R}^{N}\right)$ and $f$ is anti-symmetric with respect to the variables $x_{1}, \cdots, x_{m}$; that is, $f$ verifies (3.5). Then there exists $g \in C\left(\mathbb{R}^{N}\right)$ symmetric with respect to the variables $x_{1}, \cdots, x_{m}$ such that

$$
f(x)=x_{1} x_{2} \cdots x_{m} g(x), \forall x \in \mathbb{R}^{N} .
$$

Furthermore, if $f$ and all its derivatives up through order $m$ are bounded by Gaussian functions, then the function $g$ is also bounded by a Gaussian function.

Proof. We give the proof only in the case $m=2$, which is the case we need. The proof of the general case should be clear from this special case. 
We define the function $g$ by

$$
g(x)= \begin{cases}\frac{1}{x_{1} x_{2}} \int_{0}^{x_{2}} \int_{0}^{x_{1}} \partial_{1} \partial_{2} f\left(t_{1}, t_{2}, x_{3}, \cdots, x_{N}\right) d t_{1} d t_{2}, & x_{1} \neq 0, x_{2} \neq 0 \\ \frac{1}{x_{1}} \int_{0}^{x_{1}} \partial_{1} \partial_{2} f\left(t_{1}, 0, x_{3}, \cdots, x_{N}\right) d t_{1}, & x_{1} \neq 0, x_{2}=0 \\ \frac{1}{x_{2}} \int_{0}^{x_{2}} \partial_{1} \partial_{2} f\left(0, t_{2}, x_{3}, \cdots, x_{N}\right) d t_{2}, & x_{1}=0, x_{2} \neq 0 \\ \partial_{1} \partial_{2} f\left(0,0, x_{3}, \cdots, x_{N}\right), & x_{1}=0, x_{2}=0\end{cases}
$$

It is clear that $g \in C\left(\mathbb{R}^{N}\right)$, symmetric in $x_{1}$ and $x_{2}$, and that $f(x)=x_{1} x_{2} g(x)$.

If we now suppose that $f$ and all its derivatives up through order $m$ are bounded by Gaussian functions in $\mathbb{R}^{N}$, then (also using the symmetry properties of $f$ and its derivatives) it follows that $g$ can also be written as

$$
g(x)= \begin{cases}\frac{1}{x_{1} x_{2}} \int_{x_{2}}^{\infty} \int_{x_{1}}^{\infty} \partial_{1} \partial_{2} f\left(t_{1}, t_{2}, x_{3}, \cdots, x_{N}\right) d t_{1} d t_{2}, & x_{1} \neq 0, x_{2} \neq 0 \\ -\frac{1}{x_{1}} \int_{x_{1}}^{\infty} \partial_{1} \partial_{2} f\left(t_{1}, 0, x_{3}, \cdots, x_{N}\right) d t_{1}, & x_{1} \neq 0, x_{2}=0 \\ -\frac{1}{x_{2}} \int_{x_{2}}^{\infty} \partial_{1} \partial_{2} f\left(0, t_{2}, x_{3}, \cdots, x_{N}\right) d t_{2}, & x_{1}=0, x_{2} \neq 0 \\ \partial_{1} \partial_{2} f\left(0,0, x_{3}, \cdots, x_{N}\right), & x_{1}=0, x_{2}=0\end{cases}
$$

In addition, note that

$$
\int_{-\infty}^{\infty} \partial_{1} \partial_{2} f\left(t_{1}, t_{2}, x_{3}, \cdots, x_{N}\right) d t_{1}=\int_{-\infty}^{\infty} \partial_{1} \partial_{2} f\left(t_{1}, t_{2}, x_{3}, \cdots, x_{N}\right) d t_{2}=0,
$$

since $\partial_{1} f$ and $\partial_{2} f$ are bounded by Gaussian functions. It now follows easily that $g$ is bounded by a Gaussian function.

Proof of Theorem 1.3. Let $\theta(x)=\mathrm{e}^{|x|^{2} / 4}$. By Theorem 1 in [8], there exists a function $f \in C_{0}\left(\mathbb{R}^{N}\right) \cap L^{2}(\theta d x) \cap H^{1}\left(\mathbb{R}^{N}, d x\right)$, anti-symmetric in $x_{1}$ and $x_{2}$, verifying (1.8) with $m=2$, such that the maximal solution $u:\left[0, T_{\max }\right) \rightarrow C_{0}\left(\mathbb{R}^{N}\right)$ of the integral equation (1.1) with initial value $u_{0}=f$ is global in time. It is clear that for all $t>0$, the solution $u(t, \cdot)$ remains anti-symmetric in $x_{1}$ and $x_{2}$. (This was shown in [8.) Also, since $\alpha<4 /(N-2)$ (the Sobolev critical index), (1.1) is well posed in $H^{1}\left(\mathbb{R}^{N}, d x\right)$, and so the solution remains in that space for all $t>0$.

There is no guarantee, however, that $f$ is bounded by a multiple of $\left|\partial_{1} \partial_{2} G_{t_{0}}\right|$ for some $t_{0}>0$. We will show this to be true if $f$ is replaced by $u(t)$ with $t>0$. If $t>0$ is small enough, then condition (1.8) is also preserved.

To carry out this idea, we first look at the solution $u$ transformed using selfsimilar variables. This is a standard technique and was used in particular in the papers 3, 8. Hence our discussion will be brief. We set

$$
v(s, y)=(1+t)^{1 / \alpha} u(t, x) \text {, where } s=\log (1+t) \text { and } y=x / \sqrt{1+t} .
$$

It follows that $v(0)=u(0)=f$ and that $v$ satisfies the equation

$$
v_{s}=-L v+\frac{1}{\alpha} v+|v|^{\alpha} v
$$

The operator $L$ is given by

$$
L v=-\Delta v-\frac{1}{2} y \cdot \nabla v
$$

and is a positive self-adjoint operator on $L^{2}(\theta d x)$, with domain $H^{2}(\theta d x)$. The semigroup $\mathrm{e}^{-s L}$ is a contraction on all $L^{q}(\theta d x)$ (see [13, Corollary 2.2, p. 430]), and so by the Stein interpolation theorem (see for example Chapter III in [19]) the semigroup $\mathrm{e}^{-s L}$ is an analytic semigroup on each $L^{q}(\theta d x), 1<q<\infty$. The domain of the operator $L$ in $L^{q}(\theta d x)$ is $H^{2, q}(\theta d x)$. See [11, Theorem 2, p. 890]. 
As remarked in [8], using the abstract theory of [20, if $\alpha<2 / N$, the integral equation corresponding to (5.7) is locally well posed in all the spaces $L^{q}(\theta d x)$, $q>1+2 / N$. Since $f \in C_{0}\left(\mathbb{R}^{N}\right) \cap L^{2}(\theta d x)$, it follows that $f \in L^{q}(\theta d x)$ for all $2 \leq q<\infty$. For each such $q$, i.e. $q>1+2 / N$ and $2 \leq q<\infty$, the solution $v(s)$ is in the domain of $L$ on $L^{q}(\theta d x)$, i.e. $H^{2, q}(\theta d x)$, for all $s>0$. To see this, we first note that by Proposition 1.2 in [20, $v(s)$ is Hölder continuous into $s$ in $L^{q}(\theta d x)$ on any interval of the form $[\epsilon, S]$. The same is therefore true for the function $\frac{1}{\alpha} v(s)+|v(s)|^{\alpha} v(s)$. That $v(s)$ is in the domain of the generator of the semigroup now follows from standard semigroup theory. See, for example, 12, Theorem 1.27, p. 491].

In other words, $v(s)$ and its spatial derivatives up through order 2 are all in $L^{q}(\theta d x)$, for $s>0$. This implies that, for some sufficiently small $\gamma>0$, perhaps depending on $q$, the function $\mathrm{e}^{\gamma|\cdot|^{2}} v(s)$ is in $H^{2, q}\left(\mathbb{R}^{N}, d x\right)$ (i.e. with Lebesgue measure). Choosing $q<\infty$ large enough so that by the Sobolev embeddings we have that $H^{2, q}\left(\mathbb{R}^{N}, d x\right)$ is embedded in $C_{0}\left(\mathbb{R}^{N}\right)$, we see that, for any fixed $s>0$, the function $\mathrm{e}^{\gamma|\cdot|^{2}} v(s)$ is in $C_{0}\left(\mathbb{R}^{N}\right)$, and so $v(s)$ is bounded by some Gaussian function, $|v(s, y)| \leq C \mathrm{e}^{-\gamma|y|^{2}}$.

We return to the solution $u(t)$ in the original variables. It follows from the above considerations that for each $t>0, u(t)$ is bounded by some Gaussian function. Next we apply Proposition [5.1] where we consider $u\left(t_{0}\right)$ as the initial value, for arbitrary $t_{0}>0$. It therefore follows that, for any fixed $t>0, u(t)$ and all its partial derivatives up through order 2 are bounded by Gaussian functions. We now replace $f$ by an appropriate $u(t)$, for $t>0$ small. By the well posedness of the equation (5.7) in the $L^{q}(\theta d x)$ spaces, it follows that condition (1.8) with $m=2$ is still true with this new $f$, as long as $t>0$ is small enough.

Finally, Lemma 5.2 implies that $f(x)=x_{1} x_{2} g(x)$ for some continuous function $g$ which is bounded by a Gaussian function. This implies that $f$ is bounded by a multiple of $\left|\partial_{1} \partial_{2} G_{t_{0}}\right|$ for some $t_{0}>0$, and completes the proof of the theorem.

\section{Appendix: Symmetry decomposition of Functions}

The purpose of this appendix is to give a decomposition of an arbitrary function of $N$ variables into the sum of functions, each of which is either symmetric or anti-symmetric with respect to each of the variables. This extends the notion that a function of one variable can be expressed as the sum of an even and an odd function. While it is possible that the decomposition given here is known, the version we present is due to Robert Weissler [24.

For $1 \leq j \leq N$, let $T_{j}$ be the operator on functions of $N$ variables defined by $\left[T_{j} f\right]\left(x_{1}, \cdots, x_{j-1}, x_{j}, x_{j+1}, \cdots, x_{N}\right)=f\left(x_{1}, \cdots, x_{j-1},-x_{j}, x_{j+1}, \cdots, x_{N}\right)$. If $U=\left\{j_{1}, \ldots, j_{k}\right\} \subset\{1,2,3, \ldots, N\}$, we let

$$
T_{U} f=T_{j_{1}} \ldots T_{j_{k}} f
$$

Of course, if $U$ is the empty set, then $T_{U} f=f$. For notational simplicity, we set $S=\{1,2,3, \ldots, N\}$.

Claim 1.

$$
f=\frac{1}{2^{N}} \sum_{V \subset S} \sum_{U \subset S}(-1)^{|V \cap U|} T_{U} f,
$$

where $|V \cap U|$ denotes the number of elements in $V \cap U$. 
Proof. First consider the contribution to the sum where $U$ is the empty set. Since in this case $T_{U} f=f$, and $|V \cap U|=0$ for $V \subset S$, this contribution is precisely

$$
\frac{1}{2^{N}} \sum_{V \subset S} f \text {. }
$$

This contribution is clearly equal to $f$ since there are $2^{N}$ subsets of $S$.

Thus, we need to show that all the other contributions add up to 0. Fix any nonempty subset $U \subset S$. The resulting contribution to the overall sum is

$$
\frac{1}{2^{N}}\left(\sum_{V \subset S}(-1)^{|V \cap U|}\right) T_{U} f
$$

In fact, $\sum_{V \subset S}(-1)^{|V \cap U|}=0$. To see this, fix some element $k \in U$, which is possible since $U$ is nonempty. For a subset $V \subset S$ which does not contain the element $k$, let $\bar{V}=V \cup\{k\}$. In particular, if $k \notin V$, then (since $k \in U$ ) the sets $V \cap U$ and $\bar{V} \cap U$ differ by one element. It follows that

$$
\sum_{V \subset S}(-1)^{|V \cap U|}=\sum_{V \subset S, k \notin V}\left[(-1)^{|V \cap U|}+(-1)^{|\bar{V} \cap U|}=0\right] .
$$

This shows that all the other contributions add up to 0 and proves the claim.

Now fix a subset $V \subset S$ and set

$$
f_{V}=\sum_{U \subset S}(-1)^{|V \cap U|} T_{U} f
$$

so that

$$
f=\frac{1}{2^{N}} \sum_{V \subset S} f_{V}
$$

Claim 2. $f_{V}$ is symmetric in $x_{j}$, i.e. $T_{j} f_{V}=f_{V}$, if and only if $j \notin V$. If $j \in V$, then $T_{j} f_{V}=-f_{V}$. In particular, if $V=S$, then $f_{V}$ is anti-symmetric in all the variables, and if $V$ is the empty set, then $f_{V}$ is symmetric in all the variables.

Proof. Fix $j \in S$. We define the following map $\mathcal{R}$ between subsets of $S$ :

$$
\begin{aligned}
& \mathcal{R}(U)=U \cup\{j\}, \quad j \notin U, \\
& \mathcal{R}(U)=U \backslash\{j\}, \quad j \in U .
\end{aligned}
$$

$\mathcal{R}$ is clearly a bijection on the power set of $S$. Moreover, $T_{j} T_{U}=T_{\mathcal{R}(U)}$ for all subsets $U$ of $S$. It follows that

$$
T_{j} f_{V}=T_{j} \sum_{U \subset S}(-1)^{|V \cap U|} T_{U} f=\sum_{U \subset S}(-1)^{|V \cap U|} T_{\mathcal{R}(U)} f .
$$

Now if $j \notin V$, then $V \cap U=V \cap \mathcal{R}(U)$, which proves that $T_{j} f_{V}=f_{V}$. On the other hand, if $j \in V$, then $V \cap U$ and $V \cap \mathcal{R}(U)$ differ by one element. This establishes the claim. 


\section{ACKNOWLEDGEMENT}

Part of this work was carried out while the first author was visiting the University of Paris 13. Also, part of this work was carried out while the second author was visiting the Faculty of Sciences of Tunis. The authors wish to express their gratitude to these institutions for their warm hospitality. They also thank the Laboratoire Analyse Géométrie et Applications and Laboratoire des Equations aux Derivées Partielles for their financial support. Also, the second author benefitted from a sabbatical leave (C.R.C.T.) from his home institution for the fall semester of 2011, which facilitated the completion of this paper.

\section{REFERENCES}

[1] C. Bandle and H. A. Levine, On the existence and nonexistence of global solutions of reactiondiffusion equations in sectorial domains, Transactions of the American Math. Soc., 316 (1989), 595-622. MR.937878(90c:35118)

[2] J. Bergh and J. Löfström, "Interpolation Spaces" Springer, Berlin, 1976. MR.0482275 $(58: 2349)$

[3] T. Cazenave, F. Dickstein and F. B. Weissler, Global existence and blow up for sign-changing solutions of the nonlinear heat equation, J. Differential Equations 246 (2009) 2669-2680. MR2503017(2010d:35198)

[4] F. Dickstein, Blowup stability of solutions of the nonlinear heat equation with a large life span, J. Differential Equations 223 (2006), 303-328. MR2214937 (2007a:35064)

[5] C. Fermanian Kammerer, F. Merle and H. Zaag, Stability of the blow-up profile of nonlinear heat equations from the dynamical system point of view, Math. Ann. , 317 (2000), 347-387. MR:1764243(2001d:35088)

[6] H. Fujita, On the blowing up of solutions of the Cauchy problem for $u_{t}=\Delta u+u^{1+\alpha}$, J. Fac. Sci. Univ. Tokyo Sect. I, 13 (1966), 109-124. MR0214914(35:5761)

[7] T. Ghoul, An extension of Dickstein's "small lambda" theorem for finite time blowup, Nonlinear Analysis, T.M.A., 74 (2011), 6105-6115. MR.2833380 (2012h:35183)

[8] T. Ghoul, Global existence for the nonlinear heat equation in the Fujita subcritical case with initial value having zero mean value, J. Math. Anal. Appl., 389 (2012), 562-568. MR2876521

[9] Y. Giga, S. Matsui and S. Sasayama, Blow up rate for semilinear heat equations with subcritical nonlinearity, Indiana Univ. Math. J., 53 (2004), 483-514. MR2060042 (2005g:35153)

[10] Y. Giga, S. Matsui and S. Sasayama, On blow up rate for sign-changing solutions in a convex domain, Math. Methods Appl. Sci., 27 (2004), 1771-1782. MR2087296 (2005g:35154)

[11] R. Ikehataa, M. Ishiwata and T. Suzuki, Semilinear parabolic equation in $\mathbb{R}^{N}$ associated with critical Sobolev exponent, Ann. I. H. Poincaré, Anal. non linéaire, 27 (2010), 877-900. MR2629884 (2011j:35125)

[12] T. Kato, "Perturbation Theory for Linear Operators", Springer-Verlag, New York, 1966. MR0203473(34:3324)

[13] O. Kavian, Remarks on the large time behaviour of a nonlinear diffusion equation, Ann. I. H. Poincaré, Anal. non linéaire, 4 (1987), 423-452. MR.921547 (89b:35013)

[14] H. A. Levine, Some nonexistence and instability theorems for formally parabolic equations of the form $P u_{t}=-A u+f(u)$, Arch. Ration. Mech. Anal., 51 (1973), 371-386. MR0348216 (50:714)

[15] H. A. Levine and P. Meier, The value of the critical exponent for reaction-diffusion equations in cones, Arch. Ration. Mech. Anal., 109 (1990), 73-80. MR1019170 (90k:35039)

[16] P. Meier, Blow-up of solutions of semilinear parabolic differential equations, Journal of Applied Mathematics and Physics (ZAMP), 39 (1988), 135-149. MR937698 (90h:35037)

[17] P. Quittner and Ph. Souplet, "Blow-up, Global Existence and Steady States", Birkhäuser, Basel, 2007. MR2346798 (2008f:35001)

[18] F. Ribaud, Semilinear parabolic equations with distributions as initial values, Discrete Contin. Dynam. Systems 3 (1997), 305-316. MR1444196 (97m:35127)

[19] E. M. Stein, "Topics in Harmonic Analysis Related to the Littlewood-Paley Theory", Ann. Math. Studies 63, Princeton University Press, Princeton, NJ, 1970. MR0252961(40:6176) 
[20] F. B. Weissler, Semilinear evolution equations in Banach spaces, J. Functional Analysis 32 (1979), 277-296. MR538855(80i:47091)

[21] F. B. Weissler, Local existence and nonexistence for semilinear parabolic equations in $L^{p}$, Indiana Univ. Math. J., 29 (1980), 79-102. MR554819(81c:35072)

[22] F. B. Weissler, Existence and nonexistence of global solutions for a semilinear heat equation, Israel J. Math., 38 (1981), 29-40. MR599472 (82g:35059)

[23] F. B. Weissler, Rapidly decaying solutions of an ordinary differential equation with applications to semilinear elliptic and parabolic partial differential equations, Arch. Rach. Mech. Anal., 91 (1985), 247-266. MR806004 (87h:35164b)

[24] R. C. Weissler, personal communication.

[25] J. Wu, Well-posedness of a semilinear heat equation with weak initial data, Jnl. Fourier Anal. Appl., 4 (1998), 629-642. MR.1658660(99m:35112)

Department of Mathematics, Faculty of Science of Tunis, University Tunis El MaNar, Campus Universitaire, 2092 Tunis, Tunisia

E-mail address: slim.tayachi@fst.rnu.tn

Université Paris 13, Sorbonne Paris Cité, CNRS UMR 7539 LAGA, 99, Avenue JeanBaptiste Clément, 93430 Villetaneuse, France

E-mail address: weissler@math.univ-paris13.fr 